\title{
A Physics Based Multiscale Modeling of Cavitating Flows
}

\author{
Jingsen $\mathrm{Ma}^{\mathrm{a}}$, Chao-Tsung Hsiao ${ }^{\mathrm{a}}$ and Georges L. Chahine ${ }^{\mathrm{a}}{ }_{*}$
}

\begin{abstract}
Numerical modeling of cavitating bubbly flows is challenging due to the wide range of characteristic lengths of the physics at play: from micrometers (e.g., bubble nuclei radius) to meters (e.g., propeller diameter or sheet cavity length). To address this, we present here a multiscale approach which integrates a Discrete Singularities Model (DSM) for dispersed microbubbles and a two-phase Navier Stokes solver for the bubbly medium, which includes a level set approach to describe large cavities or gaseous pockets. Inter-scale schemes are used to smoothly bridge the two transitioning subgrid DSM bubbles into larger discretized cavities. This approach is demonstrated on several problems including cavitation inception and vapor core formation in a vortex flow, sheet-to-cloud cavitation over a hydrofoil, cavitation behind a blunt body, and cavitation on a propeller. These examples highlight the capabilities of the developed multiscale model in simulating various form of cavitation.
\end{abstract}

Key words: Cavitation, Discrete Singularity Model, Level Set, Multiscale, Transition 


\section{INTRODUCTION}

Two-phase bubbly flows play an important role in many applications. For example, cavitation in turbomachinery is deleterious and can lead to loss of efficiency, generation of noise, erosion, and increase of maintenance cost [1,2]. On the contrary, cavitation in jets can be exploited to enhance the efficiency of industrial processes such as cutting, drilling, paint removal, chemical pollutants oxidation, microorganism disinfection [3-7], algae oil extraction and nano-particles synthesis [8]. In nuclear power generation, two-phase bubbly flows occur in cooling line and equipment due to boiling, and liquid holdup affects the heat transfer efficiency. In the petroleum industry, two-phase flows occur in pipelines and in oil and gas wells. More than one-half the natural gas gathered in the US is conveyed through two-phase flow lines [9]. The ability to predict two-phase flow behavior accurately is essential in improving the performance of propulsion or power systems such as marine propellers and hydropower turbines, as well as designing energy efficient processing and transporting equipment such as gas-liquid separators and pipelines.

One of the major difficulties of accurately predicting two-phase mixture flows lies in the complex multiple length scale nature of the bubbly mixture flow, typically exampled in cavitation flows which involve sharp and violent volume changes of gas/vapor. Physics at play in this kind of problems may range from micron-scale nuclei bubbles to meter-scale large gas/vapor cavities, with very complex inter-scale transformations in between [10]. For example, on a submerged hydrofoil, in low pressure region near the leading edge small nuclei are seen to grow into large bubbles, which eventually merge to form a macro scale sheet cavity. On the other hand, detached pockets can break up continuously to form clouds of bubbles of different sizes behind the sheet cavity. Similar large volume changes can also be seen in many other systems. For example, in cooling system tiny vapor bubbles can gradually coalesce into large size cavities in the pipeline and hold up the liquid. In jet propulsion systems as the water jet plunges in the water surface significant air is entrained and forms air pockets which eventually break up into small bubbles and travel downstream to contribute to the bubbly wake [11].

Fully resolved methods such as the Boundary Element Method (BEM) [12-17], FrontTracking Method [19], and Interface Tracking Methods [20,21] directly resolve individual bubble behavior and can provide fine detail at the different scales of interests with impressive results documented so far [e.g.,12-33]. However, for these fully resolved methods the smallest 
characteristic length scale of the flow (e.g., the radius of a bubble) generally requires to be resolved by at least $O(10)$ grid points [22]. Therefore, they are only suitable for either systems dominated by large-scale air/vapor cavities [34], or small-size problems containing a limited number of bubbles. These approaches are also limited to fundamental studies such as investigating mechanisms of micro-scale processes or structures [31-33], correlating subgrid relationships for larger scale models [26,27,] or deducing valuable approximations or evaluating accuracy of asymptotic models [12, 27,22].

In practice, two-phase bubbly flows usually contain a very large number of dispersed bubbles and are modeled using averaging techniques. Continuum-based approaches [35-52] are typically used in cases where bubbles are much smaller than the characteristic lengths associated with the motion of the overall mixture. In this case, the precise location and properties of individual bubbles are not directly apparent at the global mixture flow scales. The bubbles are instead considered collectively via the equivalent continuum mixture. Other approaches combining continuum and discrete particle have also been proposed (e.g., [53-56]). In these approaches the carrier fluid is modeled using standard continuum fluid methods (Eulerian approach) and a finite number of bubbles are tracked directly (Lagrangian approach). The interaction between the two phases is achieved through local ensemble averaging [53-55] of the forces between the bubbles (or particles) and the fluid or the interaction forces are applied as a locally distributed force field [56]. Successful applications of this Eulerian/Lagrangian mixed approach have been reported in both fundamental studies and industrial applications [57-60]. These Eulerian/Lagrangian coupling schemes have shown the advantage of resolving the bubble dynamics better than Eulerian/Eulerian coupling schemes. However, they are not applicable to cases where the scales associated with the gas/liquid interface are of the same order as those of the mixture flow and when the shape and description of the interface becomes essential, e.g., in sheet cavitation and vortex cavitation problems.

Since both fully resolved approaches and averaging methods have applicability limitations, a prospective solution consists of a multiscale model capable of accurately representing two-phase bubbly flows involving various relevant scales by combining different methods to address interface structures of different scales. Some efforts have been made to deal with such multi-scale bubbly flows with noticeable examples including [e.g., 61-67], however, seamless, dynamic transition from a dispersed flow and directly resolved phases has not been 
considered so far. The challenge mainly lies on how to accurately address the meso-scale physics occurring between the micro and macro interface structures [61,62]. Specifically, the transformation between micro and macro scales includes two scenarios: a) transition from dispersed subgrid microbubbles into large distinguishable cavities with discretized free surfaces and, b) transition from a large cavity into numerous micro-scale dispersed bubbles. For the latter, the "connected cell detection" procedure proposed in [66] for modeling primary atomization could be applied. In this approach, Volume of Fluid (VOF) to Lagrangian particle transformations were conducted through tagging simply connected cells of volume fraction greater than certain values to identify contiguous lumps of liquid (droplets). This method, however, requires an exhaustive cell-by-cell search at each time step and is thus computationally expensive. Alternatively, Ma et al. [68] and Hsiao et al. [11] have successfully simulated large scale hydraulic jump and jet wake problems by integrating a level set method for free surface description and a two-fluid model or a Discrete Singularities Model for small bubbles. Efficient switching between scales was realized using a free surface instability based air entrainment model rather than through exhaustive cell-by-cell searching.

The micro to macro-scale transition, i.e., the collection of the dispersed bubbles into large cavities, is more challenging both numerically and physically since the bubbles will shrink or grow, and explode and collapse violently under cavitation conditions. After the transition step, the sudden appearance of new/liquid interfaces imposes a strong disturbance in the solution domain, resulting in a source of numerical instability. One recent interesting attempt to address this issue is the so-called clustering-method proposed by Hansch et al. [67]. They suggested introducing extra "clustering" forces in the fluid cells having gas volume fraction larger than a critical value. These forces then cause an aggregation of dispersed bubble volumes till the formation of air cavities, which in turn are to be resolved by VOF. This method however relies on an arbitrary cluster force term whose connection to physical parameters is not clear yet and needs further development. Also, only qualitative agreement of their simulation results with experimental observations was reported. For all of these reasons, one of the major objectives of this paper is to introduce a deterministic, meso-scale transition scheme to allow smooth transfer from dispersed small bubbles into large cavities, and incorporate it in a general multi-scale numerical framework for bubbly flows. 
The main features of this multi-scale framework are as follows. In the macro-scale, a continuum-based phase averaged two-phase model is considered. In this scale the gas/liquid interface of large bubbles, air pockets, and cavities is resolved directly while the interaction between small bubbles and liquid is considered through the Eulerian/Lagrangian coupling scheme. In the micro-scale, the model tracks the sub-grid bubbles which cannot be resolved in the continuum approach. To bridge the micro- and macro-scale bubbles as the bubbles grow or shrink in the flow field, inter-scale bridging schemes will utilize both the Lagrangian tracking method and the Eulerian grid technique to smoothly switch between macro and micro-scales.

\section{NUMERICAL MODEL}

The sketches in Fig. 1 present the overview of various scales regarding the current multiscale two-phase flow model, which are commonly involved in a separation or cavitating bubbly flow problem, e.g., in a configuration of separated two-phase flow in an expansion:

- At the micro-scale, transport of nuclei and microbubbles, nucleation from solid surfaces, and bubble dynamics are considered. The model addresses dispersed preexisting nuclei in the liquid, nuclei originating from solid boundaries, and microbubbles resulting from cavity disintegration or bubbles breaking up from vaporous/gaseous cavities. At this scale, the model tracks the bubbles in a Lagrangian fashion.

- At the macro-scale, a two-phase continuum-based flow is solved on an Eulerian grid. At this scale gas/liquid interfaces of large bubbles, air pockets, and cavities are directly discretized and resolved through tracking the gas-liquid interfaces with a Level Set method.

- In between, an inter-scale transition scheme is used to bridge the micro and macro scales as bubbles grow or merge to form a large cavity, or as bubbles shrink or break up from a large cavity. At this scale, model transition between micro-scale and macro-scale and vice-versa is realized by using information from both Discrete Singularity Model and Level Set method. 


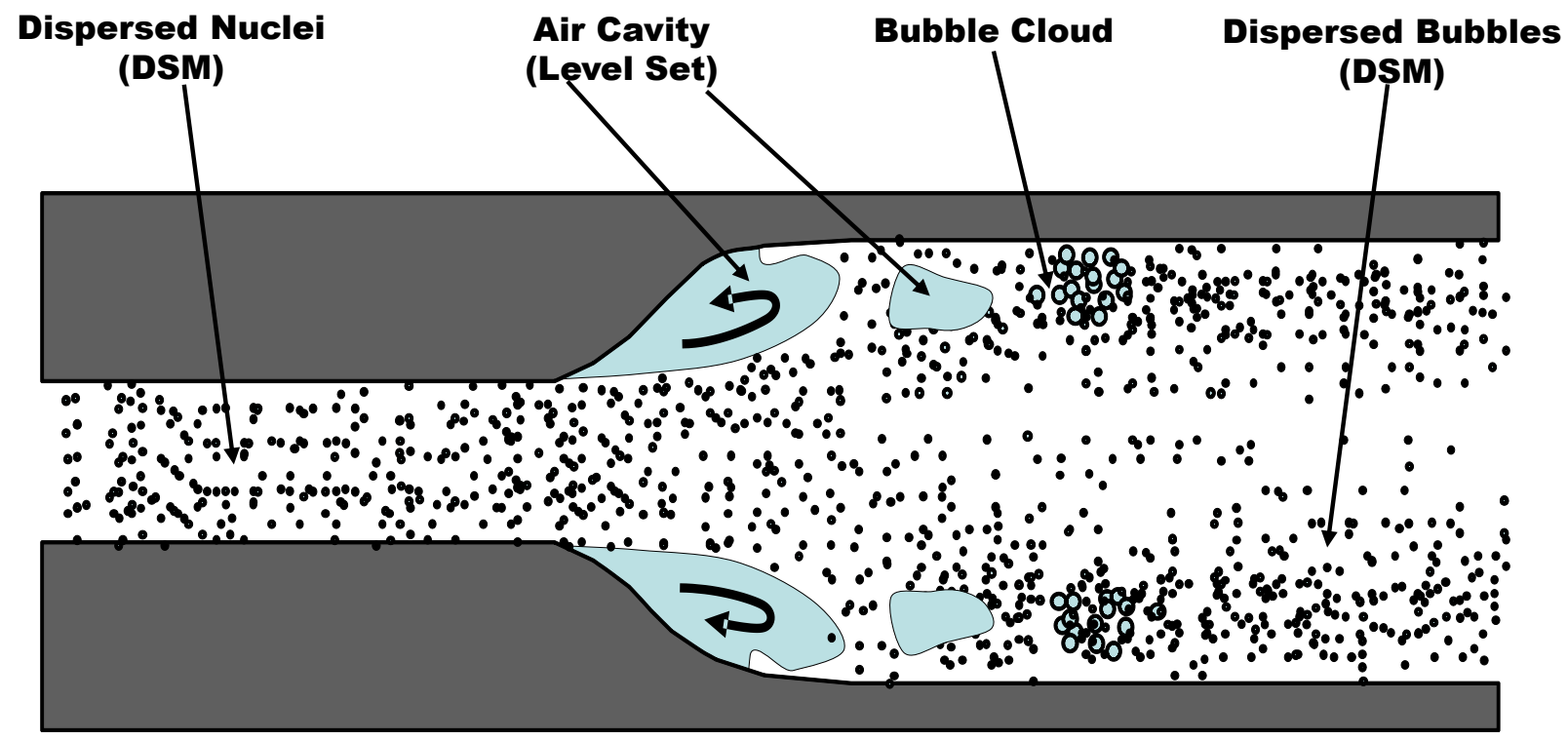

Fig. 1. Overview of the various scales involved in the multiscale two-phase flow model: Separated two-phase flow in an expansion.

\subsection{Continuum-Based Two-Phase Flow Model}

The Eulerian continuum-based model used for describing the carrier fluid phase is based on our Navier-Stokes solver, 3DYNAFS-VIS ${ }^{\odot}[70,71]$ which is an incompressible Navier-Stokes flow solver using a finite volume scheme. The continuum satisfies the following mixture NavierStokes continuity and momentum equations:

$$
\begin{gathered}
\frac{\partial \rho_{m}}{\partial t}+\nabla \cdot \rho_{m} \mathbf{u}=0 \\
\rho_{m} \frac{D \mathbf{u}}{D t}=-\nabla p+\nabla\left\{\tau_{i j}-\frac{2}{3} \mu_{m} \nabla \cdot \mathbf{u} \delta_{i j}\right\}, \\
\tau_{i j}=\mu_{m}\left(\frac{\partial u_{i}}{\partial x_{j}}+\frac{\partial u_{j}}{\partial x_{i}}\right)
\end{gathered}
$$

where the subscript $m$ refers to the mixture properties. $\mathbf{u}$ is the mixture velocity, $p$ is the pressure, and $\delta_{i j}$ is the Kronecker delta. The mixture density, $\rho_{m}$, and the mixture viscosity, $\mu_{m}$, can be expressed as functions of the void volume fraction $\alpha$ :

$$
\rho_{m}=(1-\alpha) \rho_{l}+\alpha \rho_{g}, \quad \mu_{m}=(1-\alpha) \mu_{l}+\alpha \mu_{g},
$$

where the subscript $l$ refers to the liquid and the subscript $g$ refers to the gas.

The equivalent medium has a time and space dependent density since the void fraction $\alpha$ varies in both space and time. This makes the overall flow field problem similar to a compressible flow problem. 
In our approach, which couples the continuum medium with the discrete bubbles, the mixture density is not an explicit function of the pressure through an equation of state. Instead, tracking the bubbles and knowing their concentration provides $\alpha$ and $\rho_{m}$ as functions of space and time. This is achieved by using a Gaussian distribution scheme which smoothly "spread" each bubble's volume over neighboring cells within a selected radial distance while conserving the total bubble volumes [72]. Specifically, in a fluid cell $i$, the void fraction is computed using:

$$
\alpha_{i}=\sum_{j=1}^{N_{i}} \frac{f_{i, j} V_{j}^{b}}{\sum_{k}^{N_{\text {cells }}} f_{k, j} V_{k}^{\text {cell }}}
$$

where $V_{j}^{b}$ and $V_{k}^{\text {cell }}$ are the volumes of a bubble $\mathrm{j}$ and cell $\mathrm{k}$ respectively. As described later in Section 2.3, the bubble volumes are obtained from their equivalent radius by solving a second order differential equation given as equation (13). $N_{i}$ is the number of bubbles which are influencing cell $i . N_{\text {cells }}$ is the total number of cells "influenced" by a bubble $j . f_{i, j}$ is the weight of the contribution of bubble $j$ to cell $i$ and is determined by the Gaussian distribution function. This scheme, which results in the computation of the local void fraction as the ratio of the total relevant bubble volume to the total relevant fluid volume [72], has been found to significantly increase numerical stability and to enable the handling of high-void bubbly flow simulations.

The system of equations is closed by an artificial compressibility method [73] in which a pseudo-time derivative of the pressure multiplied by an artificial-compressibility factor, $\beta$, is added to the continuity equation as

$$
\frac{1}{\beta} \frac{\partial p}{\partial \tau}+\frac{\partial \rho_{m}}{\partial t}+\nabla \cdot \rho_{m} \mathbf{u}=0
$$

As a consequence, a hyperbolic system of equations is formed and can be solved using a time marching scheme. This method can be marched in pseudo-time to reach a steady-state solution. To obtain a time-dependent solution, a Newton iterative procedure is performed at each physical time step in order to satisfy the continuity equation.

The Navier-Stokes solver uses a finite volume formulation. First-order Euler implicit differencing is applied to the time derivatives. The spatial differencing of the convective terms uses the flux-difference splitting scheme based on Roe's method [74] and van Leer's MUSCL method [75] for obtaining the firstorder and the third-order fluxes, respectively. A second-order central differencing is used for the viscous terms which are simplified using the thin-layer approximation. The flux Jacobians required in an implicit scheme are obtained numerically. The resulting system of algebraic equations is solved using the 
Discretized Newton Relaxation method [76] in which symmetric block Gauss-Seidel sub-iterations are performed before the solution is updated at each Newton iteration.

\subsection{Level Set Method for Resolved Cavities}

A level set method [77-79] is adopted to handle large bubbles and cavities because this level set method offers a flexible way to resolve large free surface deformations such as folding and breakup as compared to surface tracking methods, which require to actually discretize the interfaces.

In the level set method a smooth distance function $\varphi(x, y, z, t)$, whose zero value coincides at $t=t_{0}$ (time at which the discretized cavity is generated) with the liquid/gas interface, is defined in the whole physical domain (i.e. in both liquid and gas phases) as $d(x, y, z)$, the signed distance from the interface:

$$
\varphi\left(x, y, z, t_{0}\right)=d(x, y, z) .
$$

This function is enforced to be a material surface at each time step using:

$$
\frac{\partial \varphi}{\partial t}+u_{j} \frac{\partial \varphi}{\partial x_{j}}=0 .
$$

To avoid that the value of $\varphi$ get diffused by numerical viscosity and that the level set be distorted by the flow field, a new distance function is constructed by solving a "re-initialization equation" in pseudo time iterations [78]:

$$
\frac{\partial \varphi}{\partial \tau}=S\left(\varphi_{0}\right)[1-|\nabla \varphi|]
$$

where $\tau$ is the pseudo time, $\varphi_{0}$ is the initial distribution of $\varphi$, and $S\left(\varphi_{0}\right)$ is a sign function which is zero on the interface.

In a standard level set approach both liquid and gas phases of the fluid are solved separately using Equations (7) and (8) after identifying to which phase each cell belongs and applying appropriate interfacial boundary conditions. However, we apply here a simpler single phase level set method. A Ghost Fluid Method [77-79] is then used to impose the dynamic boundary conditions across the interface without applying any smoothing function. The dynamics boundary condition requires continuity of stresses across the surface. If the shear due to the air is neglected, the dynamic boundary conditions (balance of normal stresses and zero shears) can be written: 


$$
\begin{gathered}
p=p_{v}+\frac{g z}{\rho_{l}}+\tau_{i j} n_{i} n_{j}+\frac{\gamma \kappa}{\rho_{l}}, \quad \tau_{i j} n_{i} t_{j}^{1}=0, \quad \tau_{i j} n_{i} t_{j}^{2}=0, \\
\text { with } \tau_{i j}=\frac{\mu_{l}}{\rho_{l}}\left(\frac{\partial u_{i}}{\partial x_{j}}+\frac{\partial u_{j}}{\partial x_{i}}\right),
\end{gathered}
$$

where $p_{v}$ is the vapor pressure, $g$ is the acceleration of gravity, $\gamma$ is the surface tension parameter and $\kappa=\nabla \cdot \nabla \varphi /|\nabla \varphi|$ is the surface curvature. $\vec{n}, \vec{t}^{1}$ and $\vec{t}^{2}$ are the surface normal and two tangential unit vectors, respectively.

In this approach two ghost cells are used to impose the boundary conditions when the flow is solved at the interface cells. The interface cells for each phase are identified when one of the following six inequalities is satisfied for two adjacent cells:

$$
\varphi_{i, j, k} \varphi_{i \pm 1, j, k}<0, \quad \varphi_{i, j, k} \varphi_{i, j \pm 1, k}<0, \quad \varphi_{i, j, k} \varphi_{i, j, k \pm 1}<0 .
$$

Although the Ghost Fluid Method can help maintain a sharp interface, additional computational cost is required for the cells in the ignored phase, e.g. air. To further reduce the computational cost we apply a single-phase level-set approach in which only the liquid phase of the fluid is solved, while the cells belonging to the gas phase are deactivated during the computations. To obtain velocity information from the gas cells, we assume the following condition across the interface:

$$
\mathbf{n} \nabla u_{i}=0, \quad \mathbf{n}=\nabla \varphi /|\nabla \varphi|,
$$

which means there is no normal component of the velocity gradient at the interface, while shear could exist. With this assumption we can extend the velocities from the liquid phase into the gas along the normal direction by solving the pseudo-time iteration equation:

$$
\frac{\partial u_{i}}{\partial \tau}+\mathbf{n} \square \nabla u_{i}=0
$$

\subsection{Discrete Singularity Model for Unresolved Bubbles}

The Discrete Singularity Model (DSM), 3DYNAFS-DSM ${ }^{\circledR}$, considers all unresolved bubbles as Lagrangian singularities. It uses a Surface Average Pressure (SAP) approach [57,59,60] where fluid flow quantities are averaged along the bubble surface. This model has been shown to produce accurate results when compared to full 3D two-way interaction computations [57]. The first order term is a spherical source/sink term which represents the spherical equivalent bubble obtained by a modified Rayleigh-Plesset equation [57] to describe the bubble dynamics, 


$$
R \ddot{R}+\frac{3}{2} \dot{R}^{2}=\frac{1}{\rho}\left(p_{v}+p_{g 0}\left(\frac{R_{0}}{R}\right)^{3 k}-P_{e n c}-\frac{2 \gamma}{R}-\frac{4 \mu \dot{R}}{R}\right)+\frac{\left|\mathbf{u}_{s}\right|^{2}}{4} ; \quad \mathbf{u}_{s}=\mathbf{u}_{e n c}-\mathbf{u}_{b},
$$

where $R$ and $R_{0}$ are the bubble radii at time $t$ and $0 . \mu$ is the carrier liquid kinematic viscosity, and $\rho$ its density. $p_{g 0}$ is the initial bubble gas pressure, $k$ is the polytropic compression constant, $\mathbf{u}_{b}$ is the bubble travel velocity, while $\mathbf{u}_{e n c}$ and $P_{e n c}$ are respectively the liquid velocity and the ambient pressure "seen" by the bubble during its travel. With the SAP model, $\mathbf{u}_{\text {enc }}$ and $P_{\text {enc }}$ are respectively the averages of the liquid velocities and pressures over the bubble surface.

The second order term is a dipole representing the bubble motion. The bubble trajectory is obtained from the following motion equation:

$$
\rho_{b} \frac{d \mathbf{u}_{b}}{d t}=-\nabla p+\left(\rho_{b}-\rho\right) g+\rho\left[\frac{3}{8 R} C_{D}\left|\mathbf{u}_{s}\right|^{2}+\frac{1}{2}\left(\frac{d \mathbf{u}_{e n c}}{d t}-\frac{d \mathbf{u}_{b}}{d t}\right)+\frac{3 \dot{R}}{2 R} \mathbf{u}_{s}+6.44 R^{2} \sqrt{\rho \mu} \frac{\mathbf{u}_{s} \times \mathbf{\Omega}}{\sqrt{|\boldsymbol{\Omega}|}}\right] .
$$

The $1^{\text {st }}$ right hand side term is the pressure gradient force. The $2^{\text {nd }}$ term accounts for buoyancy. The $3^{\text {rd }}$ term is the drag force, while the $4^{\text {th }}$ and $5^{\text {th }}$ terms account for the added mass. The $6^{\text {th }}$ term is the lift force with $\boldsymbol{\Omega}$ being the deformation tensor [80].

\subsection{Transition Scheme between Micro-Scale and Macro-Scale}

The transition between microscale and macroscale models includes two scenarios:

a. transition from singularities (unresolved microbubbles) into large cavities with a tracked free surface,

b. transition from a large cavity into a set of dispersed microbubbles,

In the first scenario a), since the singularities are followed in a Lagrangian fashion and the corresponding microbubble sizes and locations are known at every time step. A criterion based on bubble size is set to "activate" the bubbles and transform them into resolved cavities using as level set field the local distances to all neighboring cells. As denoted in Fig. 2a, for each cell $i$, this distance function is determined using:

$$
\varphi_{i}=\min \left(\varphi_{L S 0}, \varphi_{b, j}\right) \quad j=1, N_{i}
$$

where $\varphi_{L S 0}$ is the distance function from previous level set computations and $\varphi_{b, j}$ is the new distance function computation using the closest distance to the newly activated bubble $j$ surface. $N_{i}$ is the number of bubbles which are "activated" around cell $i$ at the particular time step. This scheme allows a) a singularity to become a level set resolved cavity, b) multiple bubbles to 
merge together into a large cavity, and c) a single bubble to merge into a pre-existing large cavity as illustrated in Fig. 2b.

The size criterion to determine which bubble to "activate" is as following:

$$
R \geq \max \left(R_{t h r}, m_{t h r} \Delta L\right)
$$

where $\Delta L$ is the size of local grid which hosts the bubble. $R_{t h r}$ is a threshold bubble radius and $m_{t h r}$ is a threshold grid factor. This expresses that a subgrid bubble represented by singularities is switched to be represented by a level set free surface only when the bubble grows larger than the threshold bubble radius and a user selected multiple of the local grid size. The latter ensures enough grid resolution to resolve the nearly activated bubble volume. In the present study, $m_{\text {thr }}$ $=1.0$ was used. This was selected based on our previously systematic parameter-independency study [87], which showed that there is little dependency of the results on the switching criterion as long as transforming the singularity bubbles into a discretized cavity is not done too early, i.e. the criterion should ensure that the bubble has grown significantly.

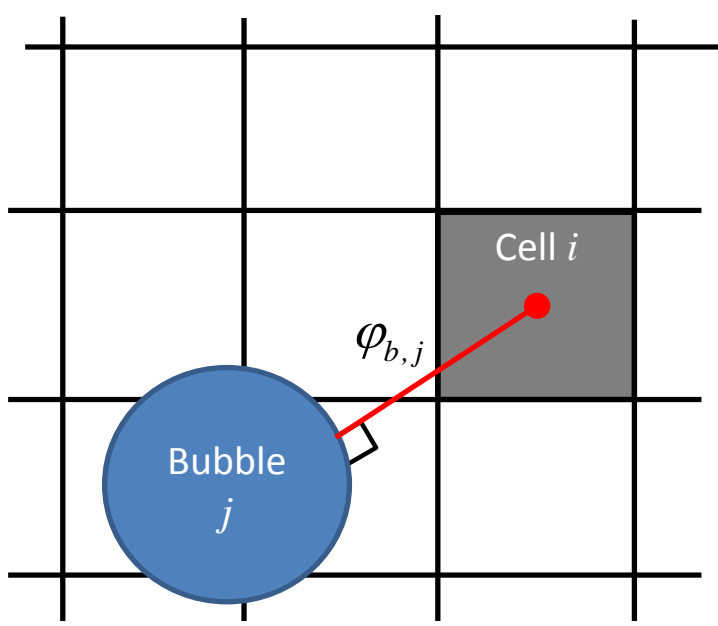

a)

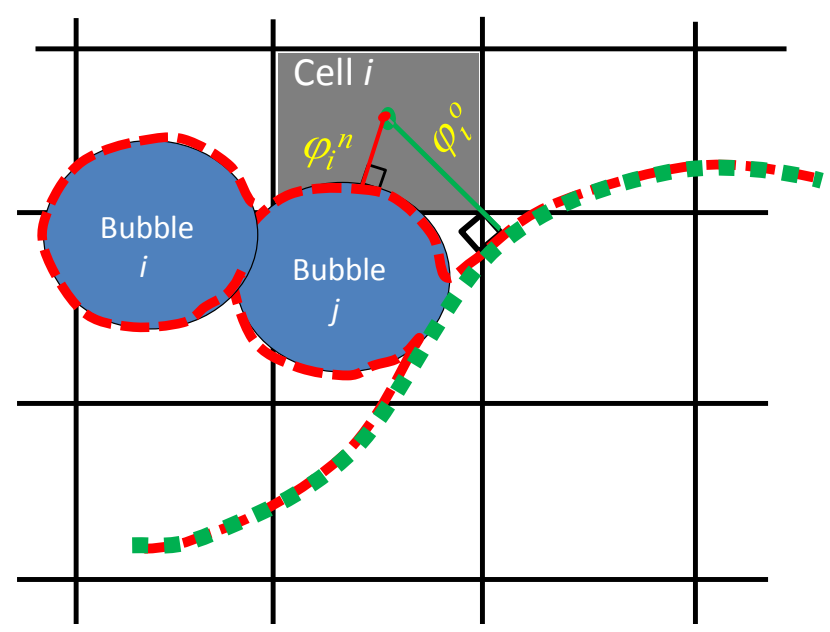

b)

Fig. 2. Illustration of the transition from DSM bubble to Level Set cavity: a) Definition of the local distance $\varphi_{b, j}$; b) Illustration of a bubble merging into a pre-existing free surface. Green dash curve is the old free-surface and the red dash curve is the new surface after the merger. $\varphi_{i}^{o}$ and $\varphi_{i}^{n}$ in are the distance function values of a cell $i$ before and after the merger.

The numerical implementation of this transition is summarized below:

At the beginning of a computation:

1. Level Set Initialization: If there are pre-existing gas-liquid interfaces in the domain, the level set value in each computation cell is computed based on its distance to the 
closest interface point. Otherwise, $\varphi \equiv \varphi_{0}$ is uniformly applied to all the cells in the domain, where $\varphi_{0}$ is a very large default value.

\section{During each physical time step:}

2. Level Set Transport: The level set transport equation, Eq. (7), is solved following standard level set methods procedures [77-79].

3. Level Set Adjustment: Each subgrid bubble represented by singularities is checked to see whether its size exceeds the threshold level of Eq. (16). If so, the level set values in the surrounding liquid cells are adjusted by comparing the original level set value and the new distances to modified cavities' interfaces following Eq. (15).

4. Level Set Re-initialization: The level set re-initialization equation, Eq. (8), is solved following standard level set methods procedures.

5. Loop: Advance to the next time step and repeat steps 2 through 4.

In the second scenario b) a collapsing cavity is identified by the zero level and its volume, tracked at each time step, determines when the cavity is collapsing. Specifically we use the zero level-set to identify the gas/liquid interface of the cavity. The volume of the cavity is tracked at each time step to determine when the cavity collapse occurs. As the cavity collapses, surface instabilities and cavity disintegration are accounted for using empirical criteria based on experimental observations. An amount of micro-bubbles of the same volume is initiated around the cavity to replace volume loss between time steps. The procedure is similar to that used to simulate bubble entrainment in breaking waves. Microbubbles are entrained into the liquid once the local normal velocity pointing towards the liquid exceeds the speed of the gas/liquid interface. This leads to a simple expression for the location and rate of bubble generation that is proportional to the liquid's local turbulent kinetic energy times the gradient of the liquid velocity in the liquid normal direction. The procedure is detailed in $[68,11]$ and not repeated here for brevity.

\section{Bubbly Flow in a Convergent-Divergent Waterjet Propulsion Nozzle}

The DSM model is coupled with the two-phase viscous flow model and firstly used to simulate bubbly flows in nozzle of pipes. Fig. 3 shows a picture from parallel experiments conducted for Navy on the subject, showing the bubbly flow inside a convergent-divergent nozzle (several other nozzle sizes and shapes were studied). It can be observed that the bubbles grow after they cross the throat and align into groups. The bottom of Fig. 3 shows a Eulerian- 
Lagrangian simulation for a mixture injection void fraction of $10 \%$. Fully coupled two-way interaction was computed and the effects of the bubble dynamics on the mixture flow were included through void fraction computed by the Gaussian Smoothing void fraction scheme in Eq. (4). Bubbly flow structuring into clusters is observed in the divergent section between the throat and the exit. Bubbles collect to low pressure regions that were convected downstream. The bubble clustering is similar to what was observed experimentally and deserves further attention. The detailed results of this study were presented in [81,82] and are not repeated for brevity purpose.
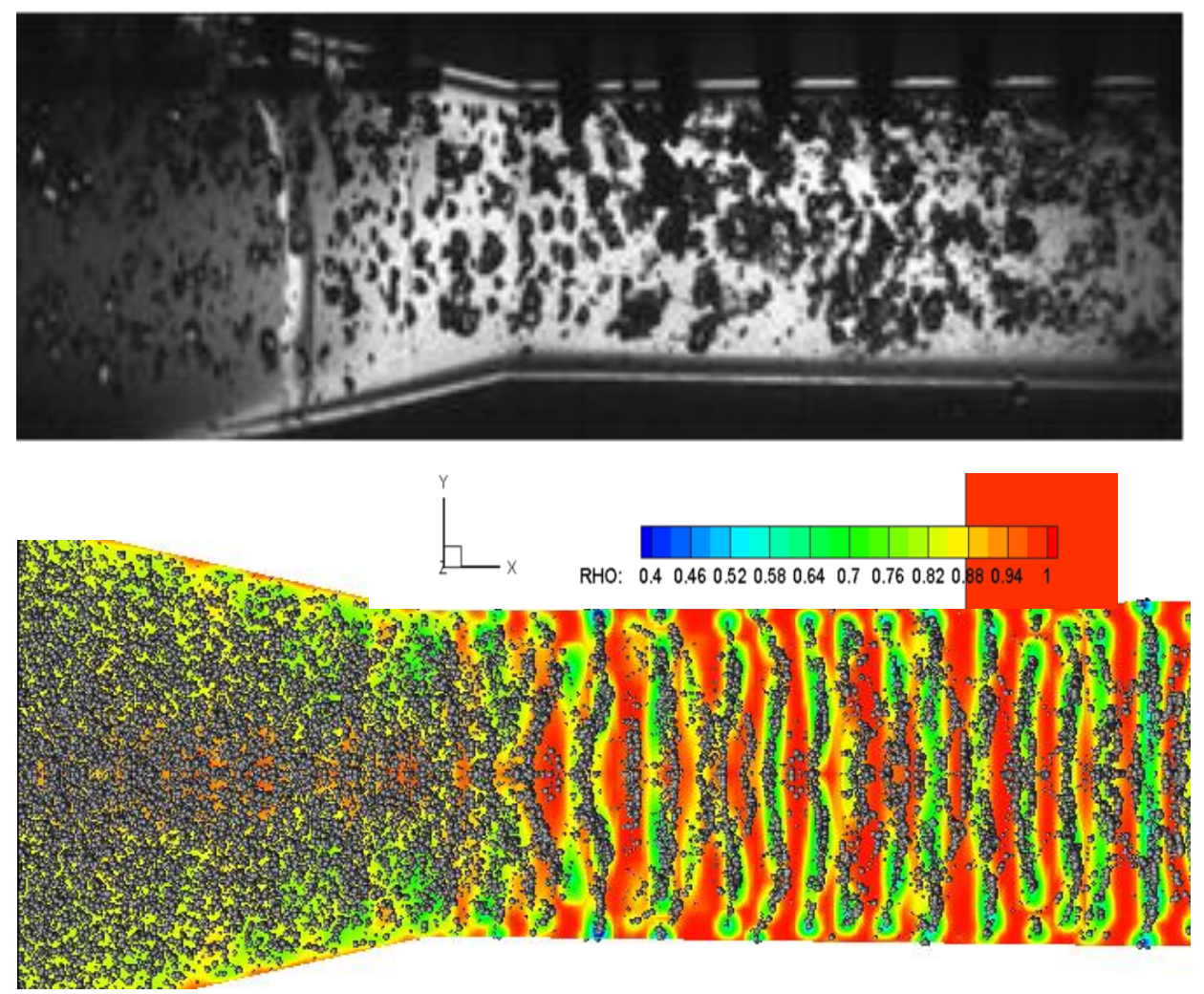

Fig. 3. Visualization of the flow inside a two-phase convergent-divergent nozzle and a two-way coupled numerical simulation using Eulerian-Lagrangian model [81,82].

\section{Bubble Capture in a Line Vortex}

The above described multiscale scheme can be illustrated by the simulation of bubble dynamics, capture, and gaseous/vaporous core formation in a Rankine line vortex. Similar problems have been studied previously by different researchers (e.g., Chahine et al. [10], Tryggvason et al [24] and Hsiao et al. [70]) using either Discrete Singularity Model (DSM) or fully resolved Direct Numerical Simulations (DNS), indicating the former is suitable when a nucleus is small while the latter is more accurate when it is large $[10,24,70]$. 


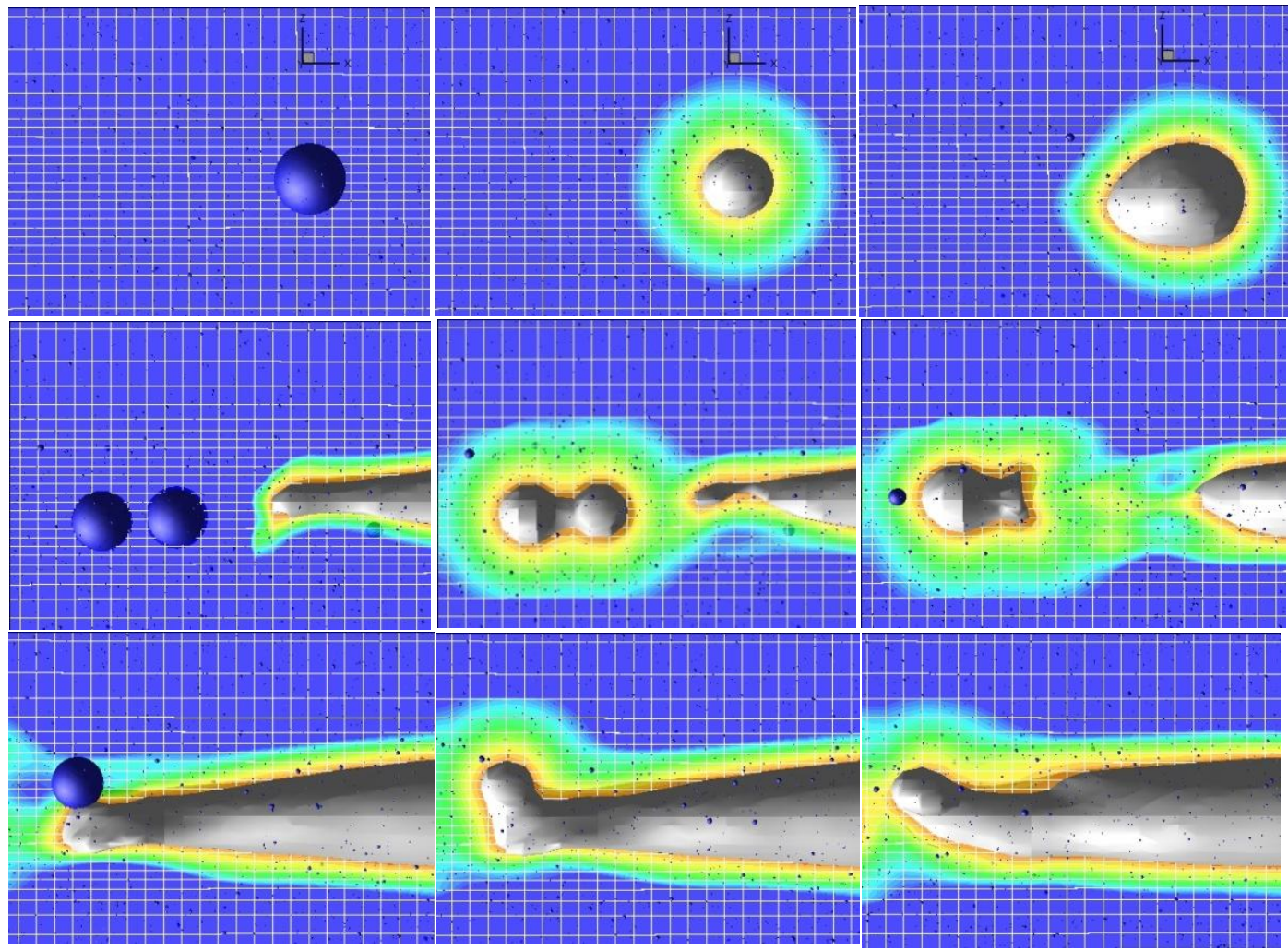

Fig. 4. Three selected sequences highlighting the capacities of capturing enriched phenomena by the multiscale method: (Top) Bubble deformation; (Middle) Bubble coalescence; (Bottom) Bubble merging into the developing vapor core. The dark blue spheres are DSM bubbles while the grey iso-surface is the liquid-vapor interface represented by level set method. The grey mesh is the computation cell used by the Eulerian solver and the background color denotes the value of level set at each cell.

A Rankine vortex is defined by the rotation velocity, $u_{\theta}$, and pressure, $p_{\omega}$, of the vortical flow given by

$$
\begin{aligned}
u_{\theta}(r)= & \left\{\frac{\Gamma}{2 \pi a_{c}^{2}} r, r \leq a_{c} ; \quad \frac{\Gamma}{2 \pi r}, r>a_{c}\right\}, \\
& p_{\omega}(r)=\left\{\begin{array}{l}
p_{\infty}-\frac{\rho \Gamma^{2}}{4 \pi^{2} a_{c}^{2}}+\frac{\rho \Gamma^{2} r^{2}}{8 \pi^{2} a_{c}^{4}}, r \leq a_{c} \\
p_{\infty}-\frac{\rho \Gamma^{2}}{8 \pi^{2} r^{2}}, r>a_{c}
\end{array}\right\} .
\end{aligned}
$$

Here, $r$ is the radial coordinate, $\Gamma$ is the circulation strength and $a_{c}$ is the viscous core radius. 


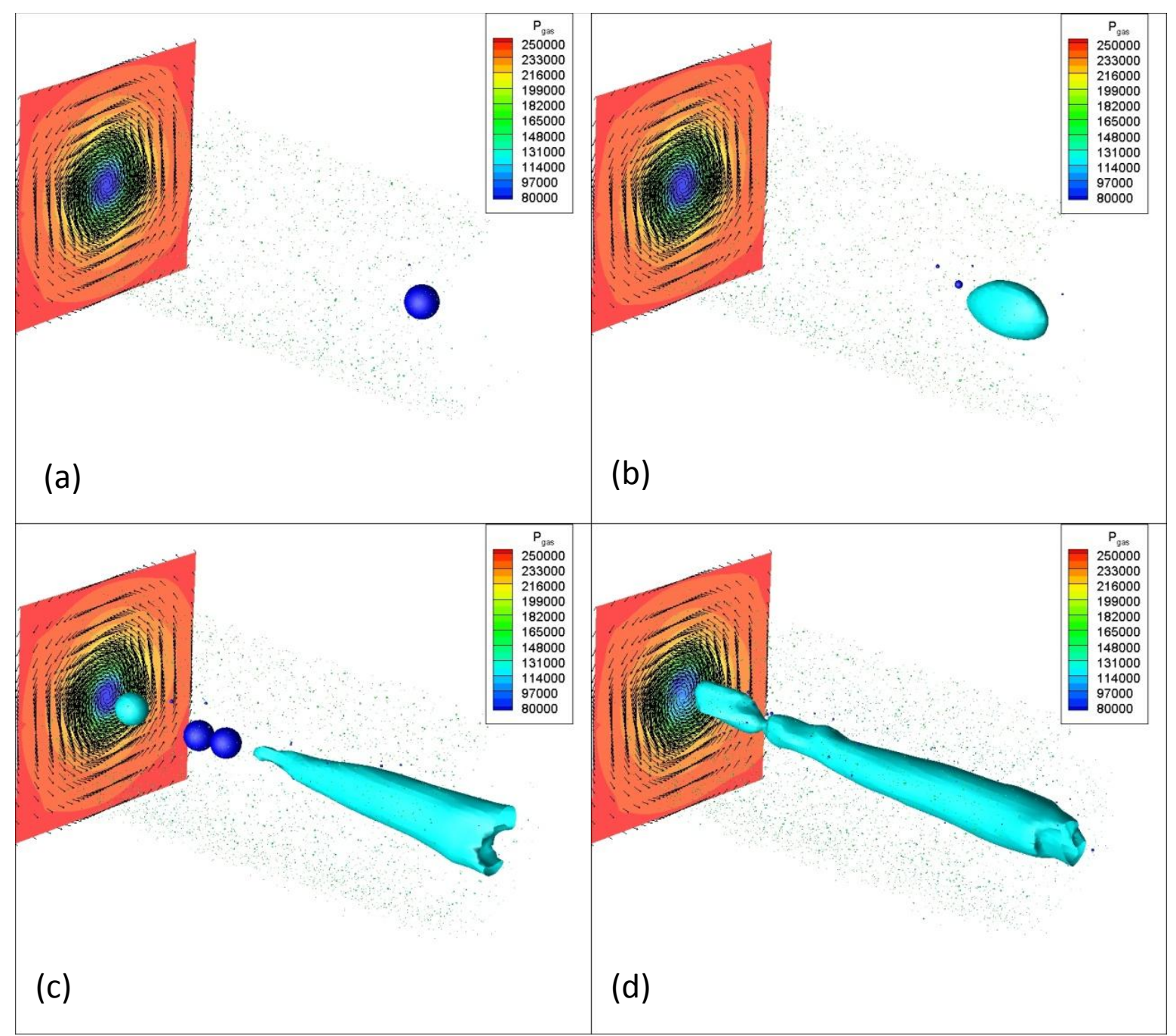

Fig. 5. Modeling of the two-phase flow inside a Rankine Vortex. Time sequence of the development of a cavitation core on the axis of the vortex.

In the illustrations below, the vortex is selected to have a viscous core radius $a_{c}=5 \mathrm{~mm}$ and a circulation $\Gamma=1.255 \mathrm{~m}^{2} / \mathrm{s}$. A distribution of bubble nuclei with sizes between 20 and $100 \mu \mathrm{m}$ and an initial void fraction $\alpha=10^{-4}$, as shown in Fig. 5, is tangentially released between radial distances from the vortex axis, $R_{\text {release }}=3.5 a_{c}$ and $3 a_{c}$.

The radii of the released nuclei are such that all are initially of subgrid size and are therefore modeled as discrete singularities (DSM). After tangentially entering into the vortex flow, they gradually travel towards the vortex core center as driven by the pressure gradient which exerts forces on the bubbles pointing inward. At the same time the bubble sizes increase due to the drop of encountered pressure. The bubbles are modelled by DSM until they grow to be large enough and then they are switched to be represented by the level set method. This is 
highlighted in Fig. 4, which is a side view displaying three selected sequences during the gas/vapor core formation and evolution inside the vortex. The top row in Fig. 4 shows the initialization of level set field by the first cavitating bubble. That is to say, the bubble wall is replaced by the free surface corresponding to a level set value of 0 . Then the level set value in the flow filed around the bubble are computed by following the definition in Fig. 2 a. Once the bubble is represented the level set method then its deformation can be captured by solving Equation (7). From the Fig. 4 it is also seen the model captures the details of a bubble elongating along the axis of vortex, coalescence with its neighbors, and its merge into the cavity. These enriched phenomena are beyond the reach of either Discrete Singularity Model or level set method alone under the same grid resolution. As it is well known, by definition the former is not able to describe bubble deformation and coalescence without borrowing extra subgrid models, while the latter will require a mesh at least two to three orders finer than the current one in order to solve the dynamics of each nucleus. Fig. 5 shows a line vortex is formed at the center of the domain drawing bubbles to the axis to form a gas/vapor core.

\section{Simulation of Unsteady Sheet Cavitation on a NACA0015 Hydrofoil}

In this section, the present multiscale model is used to simulate sheet cavitation dynamics on a NACA0015 hydrofoil. For this simulation, a very important physical aspect of our approach is to account for nucleation not only from the free field, but also from the rigid boundaries using a wall nucleation model $[87,88]$.

\section{$5.1 \quad$ Nucleation Model}

The wall nucleation model involves the following parameters, which are functions of the wall material properties and the local flow conditions $[87,88]$ :

a) $P_{t h r}:$ a nucleation pressure threshold,

b) $N_{s}$ : a number density of nucleation sites per unit area,

c) $f_{n}:$ a nucleation rate, and

d) $R_{0}$ : the initial nuclei size(s).

At each time step the pressure on a panel on the rigid boundary is compared with $P_{t h r}$ to determine if nucleation should occur. Once the pressure drops below the threshold, the cell releases $N$ nuclei per second, $N=N_{s} f_{n} \Delta A$, where $\Delta A$ is the surface area of the grid cell. During a 
time step $N \Delta t$ nuclei are released where $\Delta t$ is the integration time step. All above parameters are functions of surface roughness and temperature. In the results shown below, $P_{t h r}$ is selected to be the vapor pressure. The initial size of the nuclei emitted is selected to be $10 \mu \mathrm{m}$ with the number of nucleation sites per unit area value selected to be $N_{s}=224 / \mathrm{cm}^{2}$ and the nucleation frequency selected to be $f_{n}=22 \mathrm{kHz}$. These parameters are chosen based on the study in $[87,88]$, where the results became independent of $N_{s}$ and $f_{n}$ when values larger than threshold values were used.

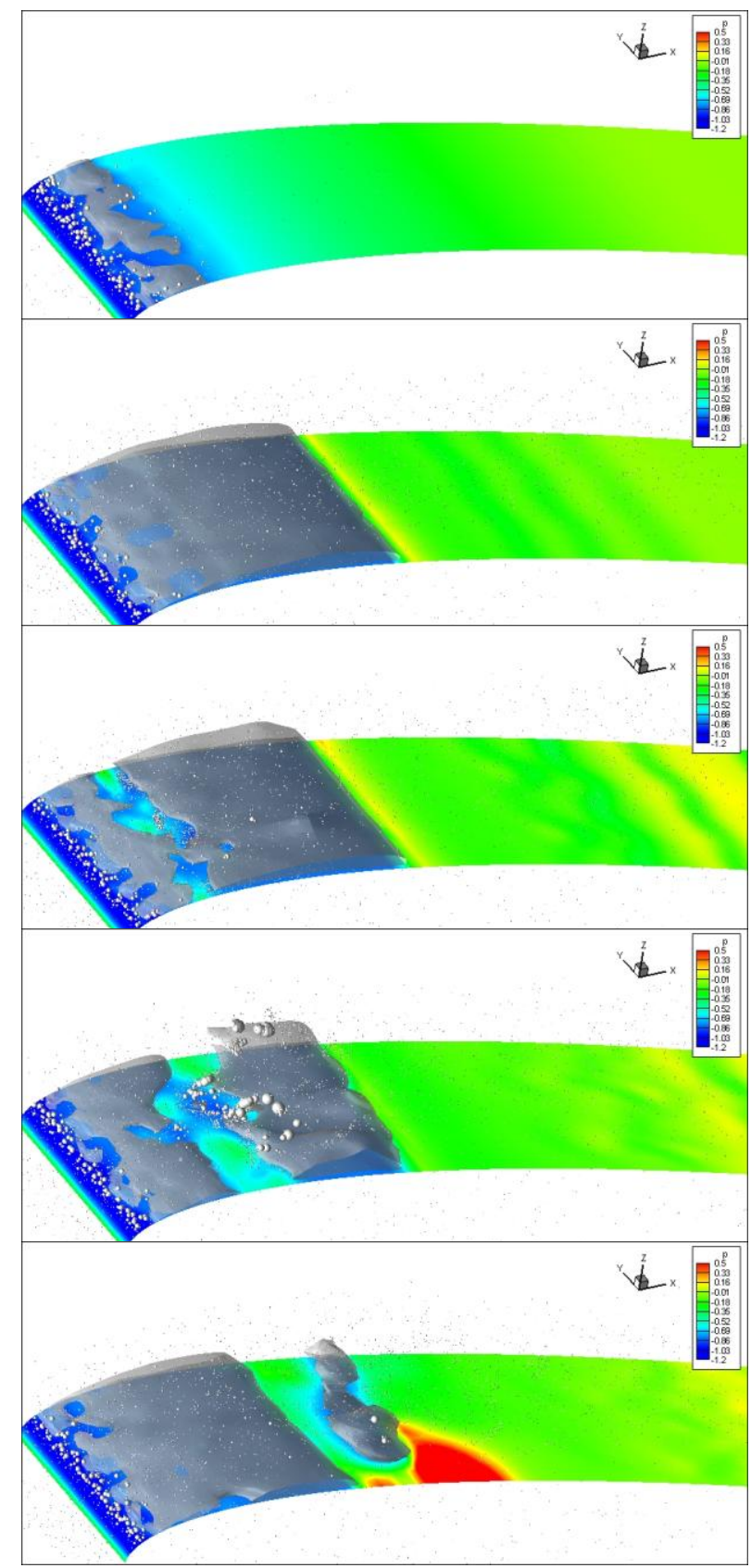

Fig. 6. An example time sequence of sheet-to-cloud cavitation simulated by the multiscale twophase flow model. $\left(\sigma=1.65, \alpha=8^{\circ}, U_{\infty}=10 \mathrm{~m} / \mathrm{s}, L=12 \mathrm{~cm}\right)$. 


\subsection{Simulation Results}

As illustrated Fig. 6, the present model successfully captures sheet cavity initiation, dynamics, and breakup on a well-tested foil. Bubbles nucleated from the blade surface and free nuclei in the liquid were the basis of the simulations and no empirical mass exchange models were needed. Cavitation initiates on the hydrofoil leading edge when the local pressure drops below the vapour pressure which is highlighted in Fig. 7. The nuclei grow, aggregate, and coalesce into a large cavity well-captured with the Level Set method. As the sheet cavity develops, its trailing edge curves into a reentrant jet, which moves upstream over the foil and into the cavity, then breaks it into a smaller cavity and a vortical large bubble cloud, which collapses generating high pressures.

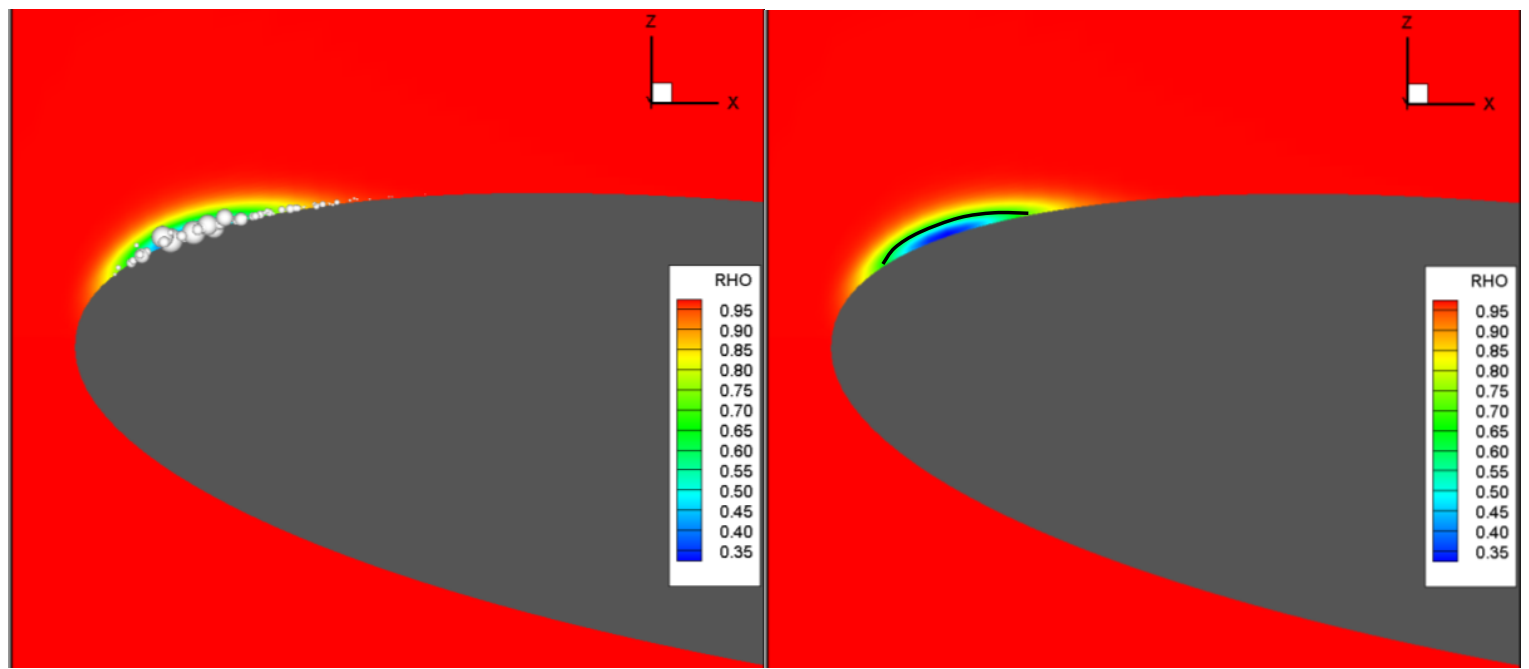

Fig. 7. A zoomed front view of cavity initiated by growing nuclei from the solid boundary at leading edge $\left(\sigma=1.65, \alpha=8^{o}, U_{\infty}=10 \mathrm{~m} / \mathrm{s}, L=12 \mathrm{~cm}\right)$. 


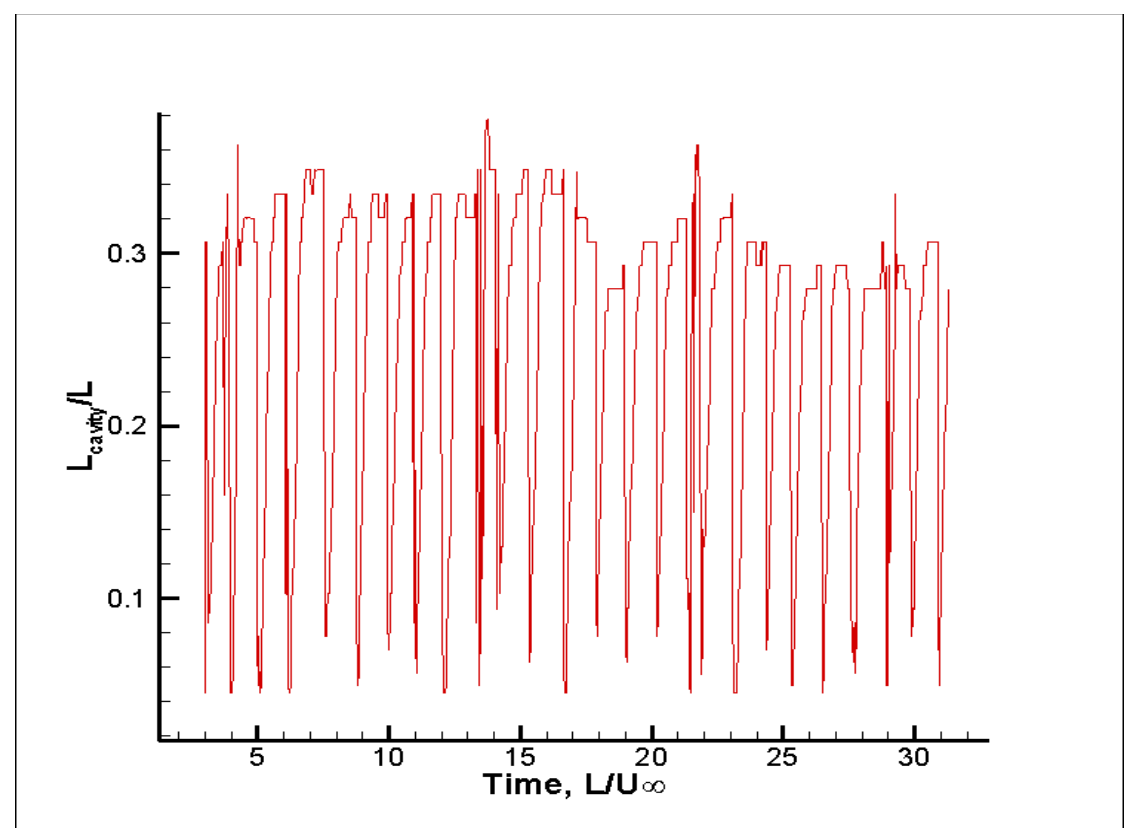

Fig. 8. Time History of Cavity Length $\left(\sigma=1.65, \alpha=8^{o}, U_{\infty}=10 \mathrm{~m} / \mathrm{s}, \mathrm{L}=12 \mathrm{~cm}\right)$.

This periodic shedding phenomenon can be further quantified by analyzing the time histories of cavity length (Fig. 8), as well as the instantaneous pressures and stream-wise velocities in the flow. Fig. 9 presents the time histories of the instantaneous pressures and streamwise velocities at three different locations close to the suction side of the foil: a) near the leading edge, b) at the trailing edge and c) at $x=0.4 L$. The last point c) is selected as it is close to the average trail location of the fully developed sheet cavity. It is seen that the oscillation of pressure and velocity are strongest at point c). On the other hand both the pressure and velocity near the trailing edge are varying around more or less the same base levels as at $x=0.4 L$ but with much smaller magnitudes. The reason for that is, for the partial cavitation occurring at this cavitation number, the cavity detachment and cloud collapse are mostly happening on the upstream half of foil surface, thus less oscillations are seen downstream. Near the leading edge, the streamwise velocity is mostly around a highest positive value while the pressure is about its minimum, similarly as usually seen on a non-cavitating wetted foil. 

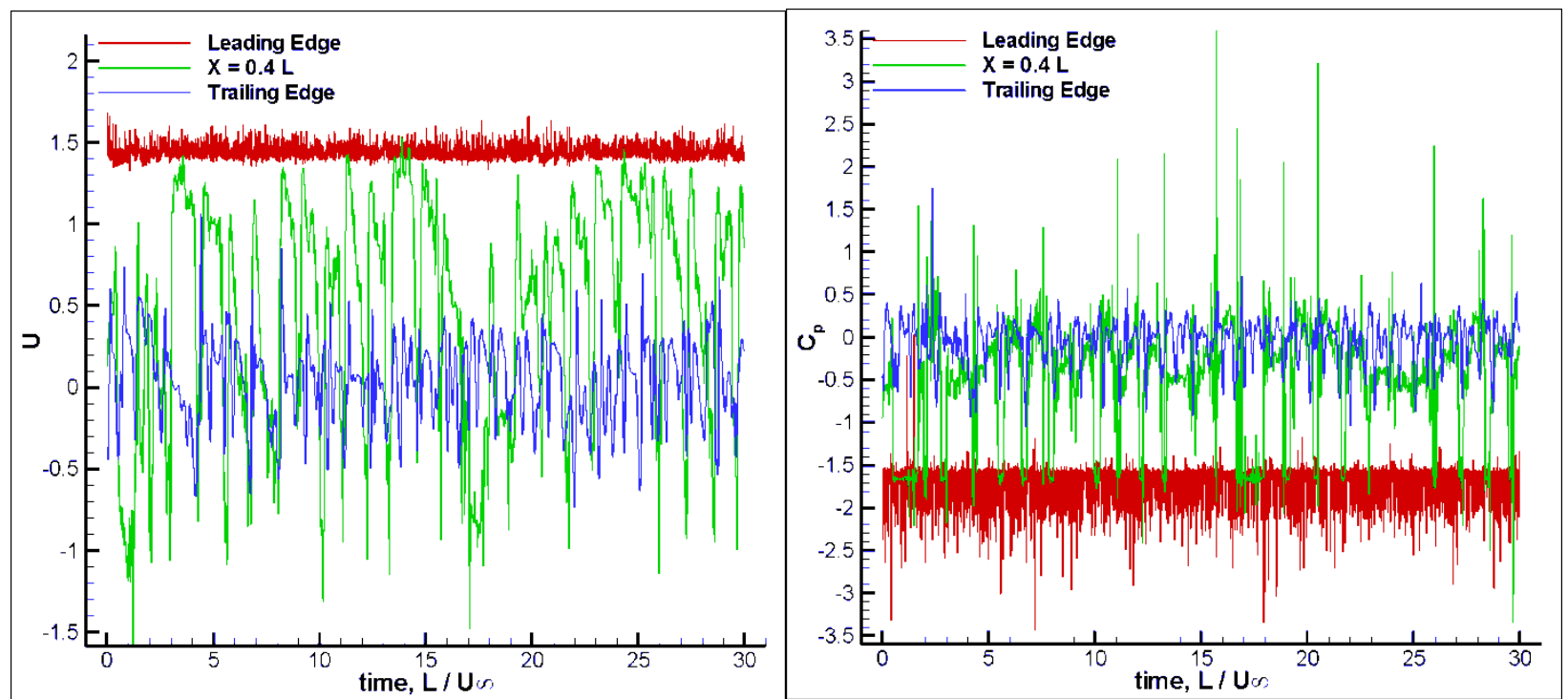

Fig. 9. Comparison of time histories of the stream wise velocity (Left), and of the instantaneous pressure (Right) monitored at three points (leading edge, trailing edge and $x=0.4 L)$ near the suction side of the foil for $\sigma=1.65$.

The location c) $x=0.4 \mathrm{~L}$ close to the closure region of the sheet cavity alternatively experiences negative (reentrant jet development) and positive stream-wise velocities with magnitudes as high as the incoming flow $U_{\infty}$. The time interval between consecutive negative velocity maxima is repeatable and has a value close to $1.3 \mathrm{~L} / U_{\infty}$. The corresponding oscillation frequency is about $0.78 U_{\infty} / L$, which matches well with the experimentally measured shedding frequency for the same cavitation number [9]. Comparison of the numerical results with experimental observations for a large range of $\sigma$ is seen to be very good, as illustrated in Fig. 10. 


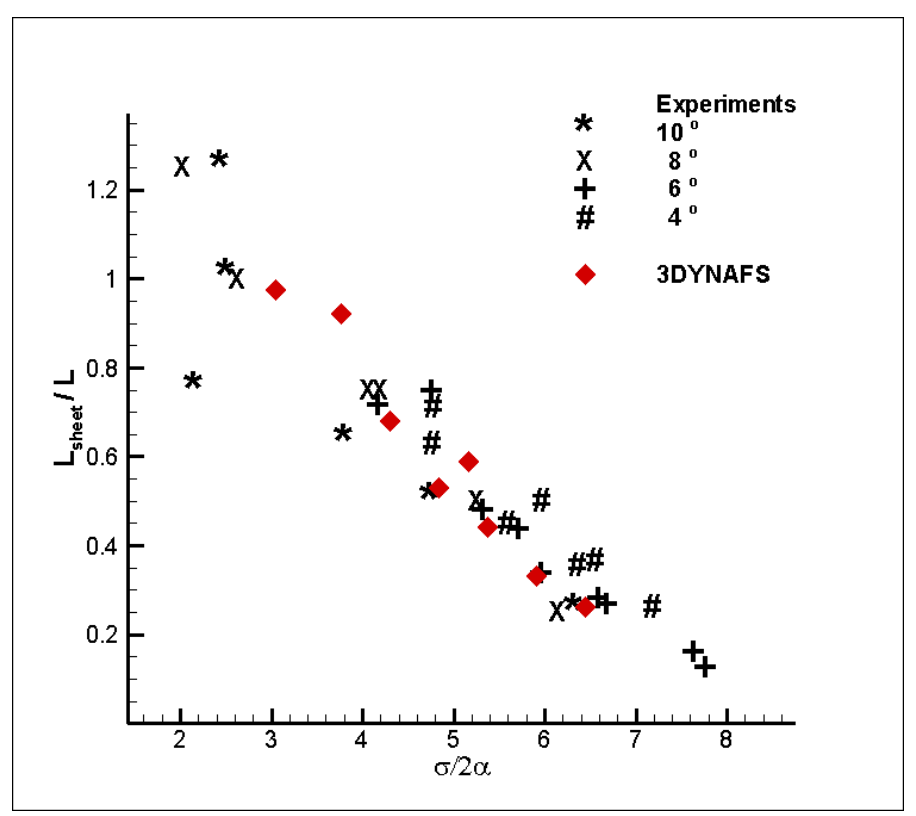

Fig. 10. Comparison of the shedding frequencies of the NACA0015 sheet cavity computed with $2 D$ simulations using the preset numerical method and the experimental results presented in reference [89].

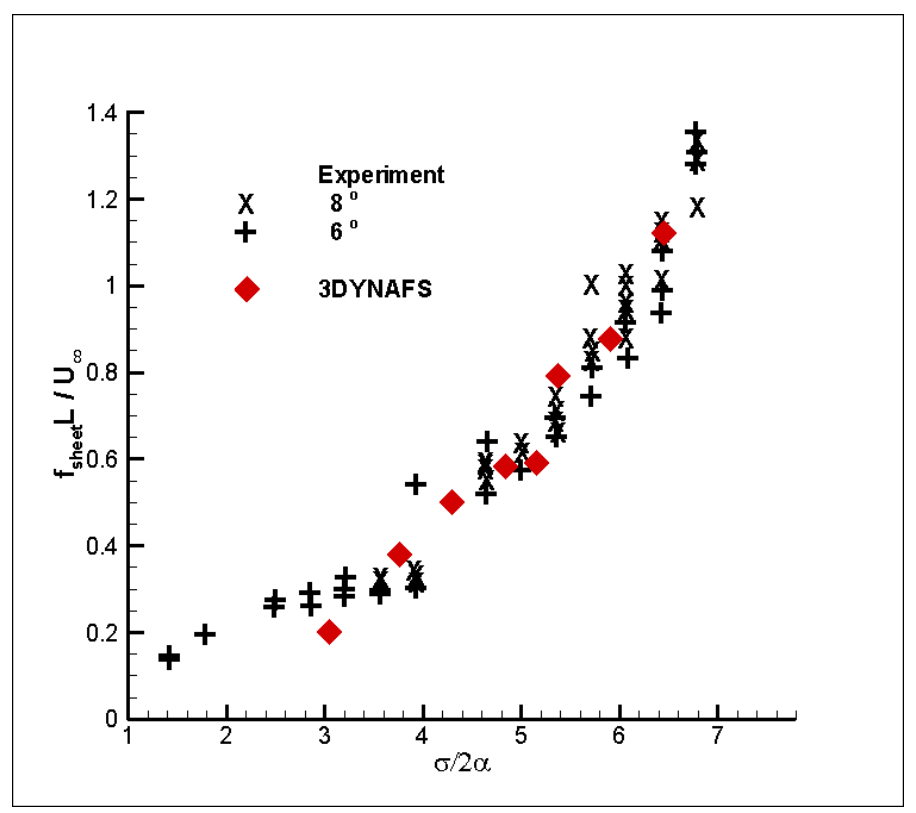

Fig. 11. Comparison of the time-averaged cavity length on the NACA0015 from the $2 D$ simulations using the preset numerical method with experimental results from [89].

Another important quantity reported experimentally for sheet cavities is the timeaveraged sheet length This is shown in Fig. 10, which illustrates that the size of the cavity increases as $\sigma$ drops. The results are also compared to those obtained experimentally and are seen to produce a good match. 
All of above shows both the shedding frequency and the time- frequency and the timeaveraged cavity length compare well with the experimental measurements over a wide cavitation number range.

\section{Cavitation behind a Blunt Body}

In this section, the method is applied to simulate the various cavitation stages behind a blunt body moving at increasing speeds. This flow was previously studied experimentally in a controlled laboratory setting [90]. Flow visualization of the supercavity was achieved by high resolution digital photography as well as by high speed photography. The observed cavitating flow behind the body at different speeds can be seen in Figure 12 which, for example, shows the evolution of the cavitating flow from limited cavitation at low flow rate till supercavitation with an increasing flow rate.
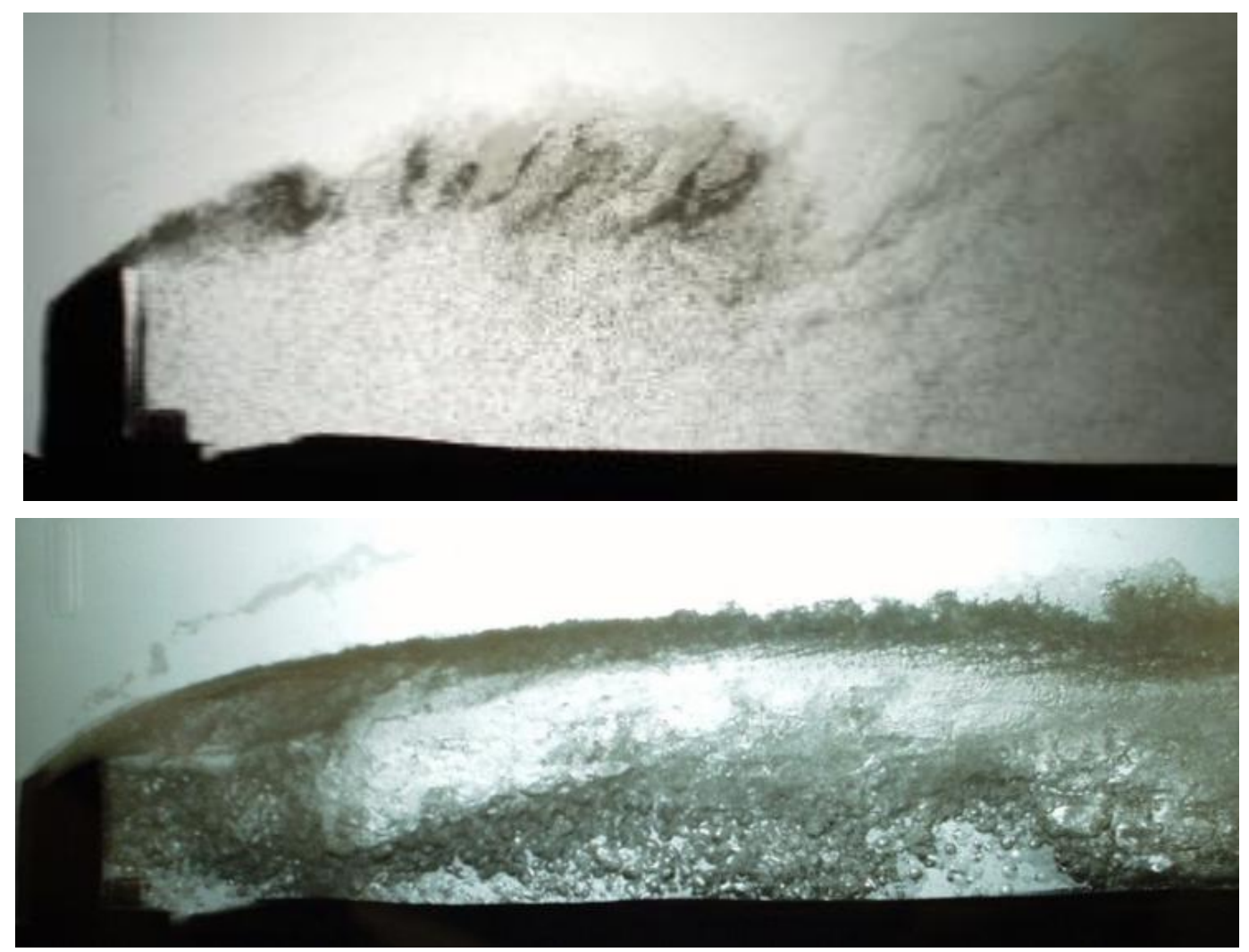

Figure 12. Pictures of the cavitating flow behind a blunt body at low (top) and high (bottom) speeds [90].

Figure 13 (top) shows the layout and dimensions of the modeled part of the test section. Three areas with different expansion profiles can be identified. A short flow entrance section with a length of 2 in, where the blunt body is mounted has a constant cross section. The test 
section then gradually expands over 30 in. to maintain near constant pressure over the cavity. Finally, a high expansion region of length $1 \mathrm{ft}$ enables the flow to slow down and the local pressure to gradually increase to reach the atmospheric pressure at the exit. The two parallel side walls are $1 \mathrm{in}$. apart, which is significantly smaller than the section length allowing to assume the flow to be two-dimensional. An 11-block mesh with 20,000 grid points was created for the simulation (Figure 13 bottom). The mesh was made finer in the regions of interest, i.e., close to the body, and then gradually became coarse downstream of and away from the body. More details about the simulation setup are available in [91].

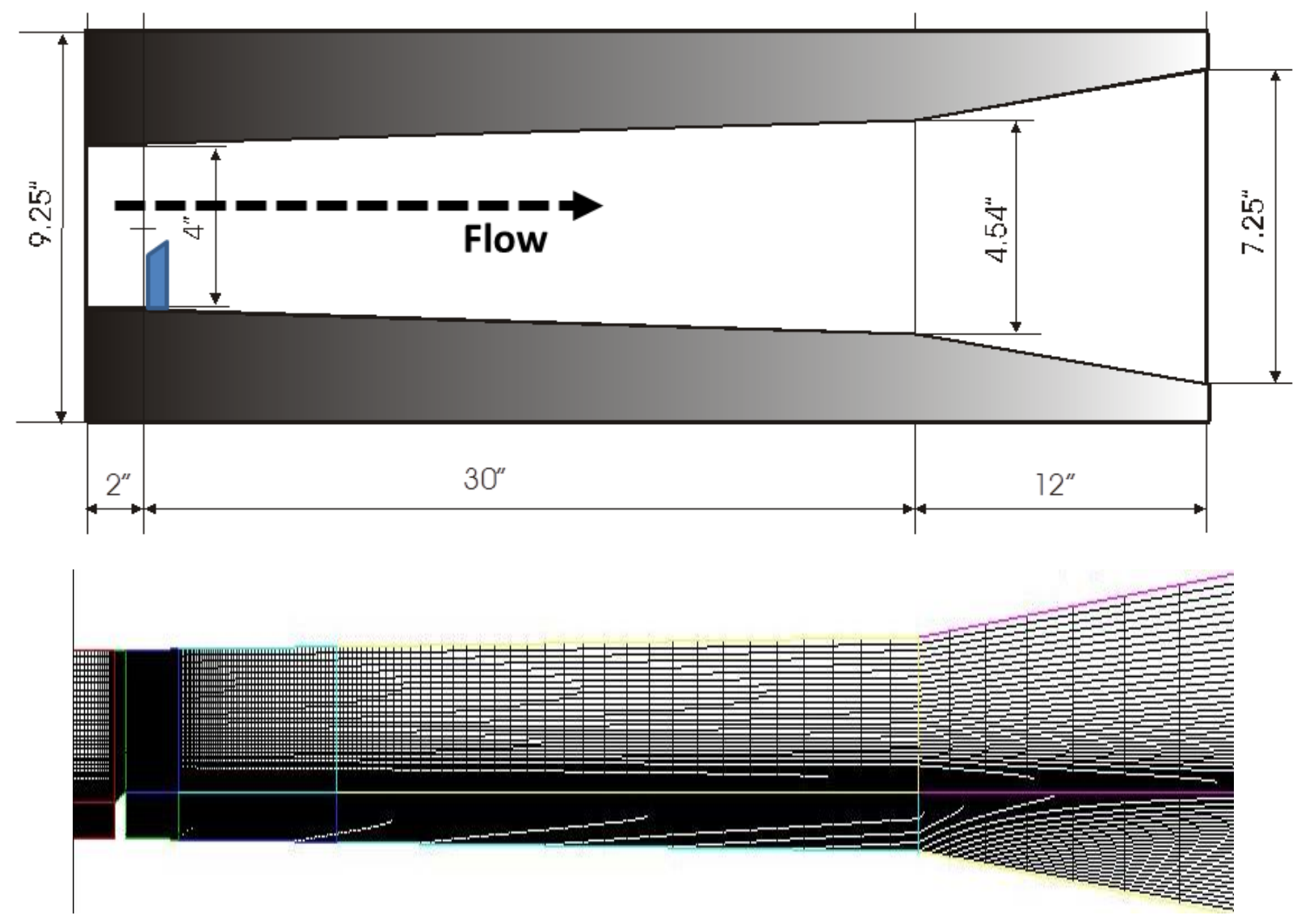

Figure 13. Setup of separated flow experiments and simulation. A) Sketch of the test section with dimension (stretched along the vertical direction) B) The $2 D$ mesh generated for simulation.

\subsection{Vortical Structures and Cavitation Inception}

Flow separation behind the blunt body results in the shedding of vortices, which capture nuclei at their centers as we have seen in above Section 4, causing them to grow and collect in the low-pressure regions. This is clearly seen in Figure 12 -top and in Figure 14. In the top 
picture in Figure 14 the flow field is shown with vorticity contours in order to highlight the large vortical structures formed behind the body. The cores of these vortical structures are low pressure regions which could lead to explosive nuclei growth and cavitation. This is evidenced by the bottom picture in the same figure, the water vapor pressure. Bubble clusters are also observed in these cores, which merge and become elongated cavities. The former are simulated by DSM bubbles while the latter are described by free surface dynamics using the level set method.

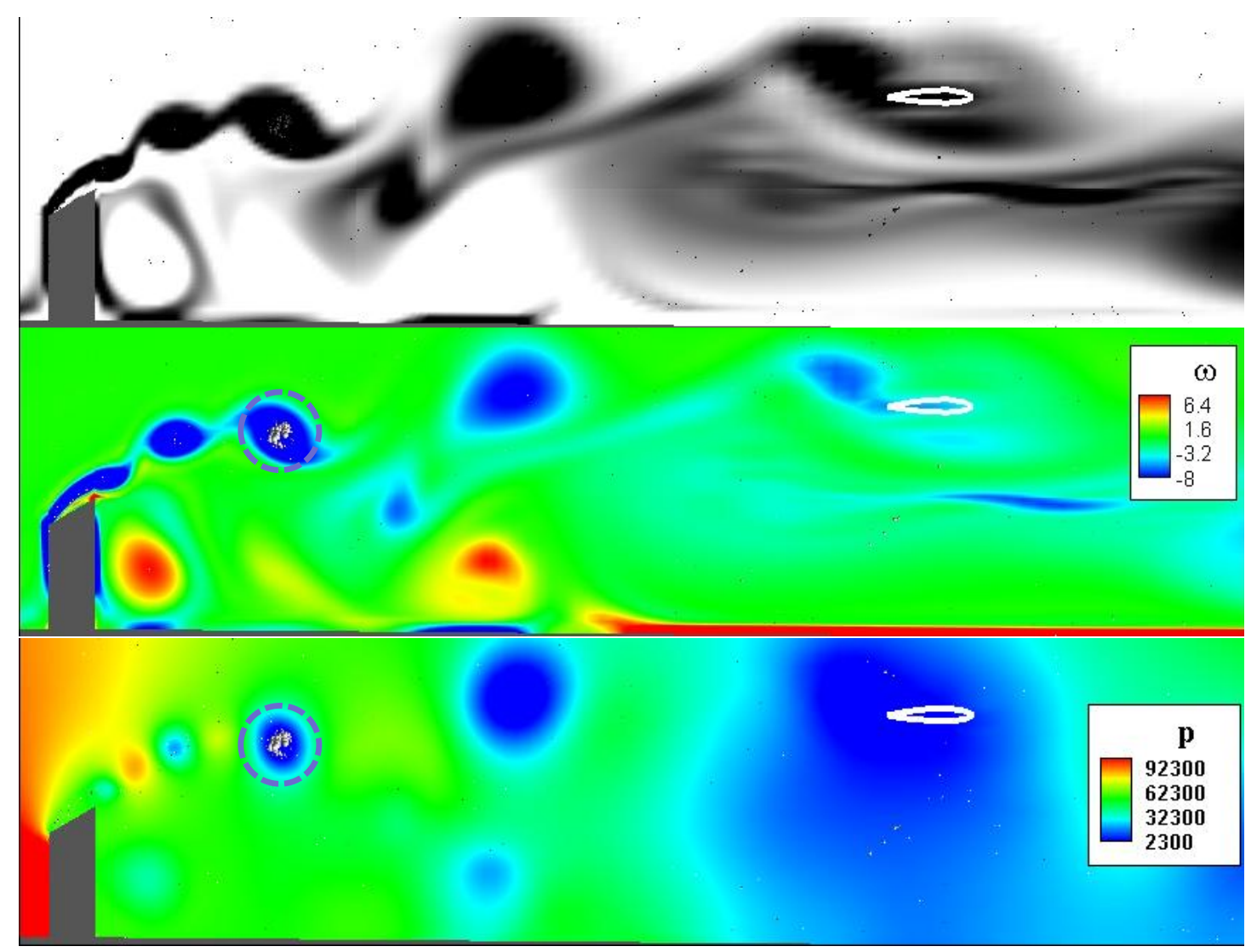

Figure 14. Top: Large vortical structures formed behind the body (Grey scale corresponds to vorticity intensity); Middle \& Bottom: Entrainment of nuclei bubbles into the low-pressure vortical centers and cavitation inception with the background color representing the vorticity intensity (Middle) and fluid pressure (Bottom), respectively, while the silver spheres are the nuclei bubbles and the white lines correspond to the zero level set cavity interface. The bubbles in the dash-circled region highlights the bubble cluster drawn to the vortex center 


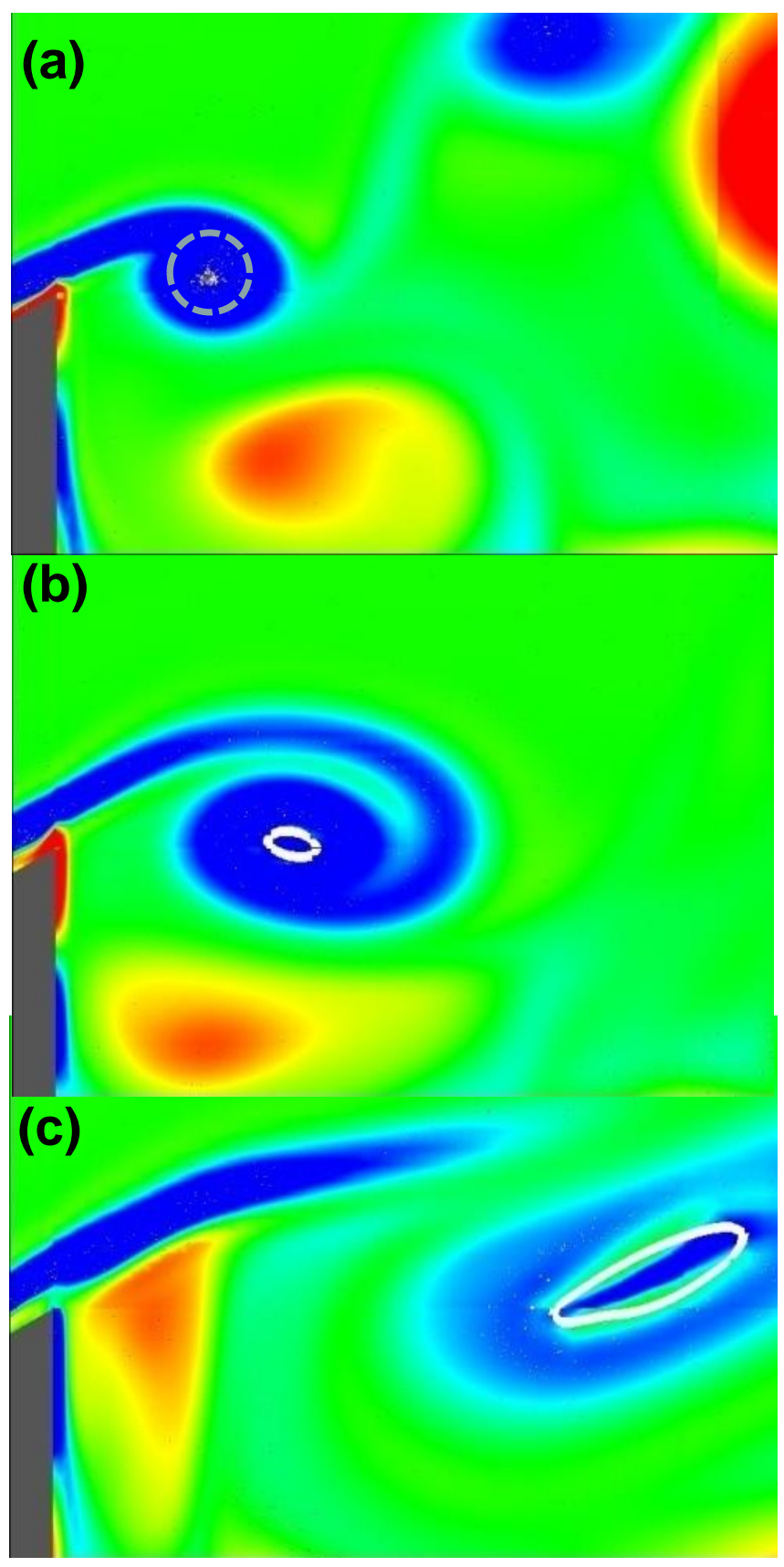

Figure 15. A time sequence of the flow behind the body, showing: (a) bubble entrainment (highlighted in the dash-circled region), (b) cavitation inception and (c) cavity elongation due to the vortical structure evolution behind the body for the $6 \mathrm{~m} / \mathrm{s}$ case. Color contours represent vorticity. Silver spheres are the nuclei bubbles and the white line corresponds to the zero level set cavity interface. 


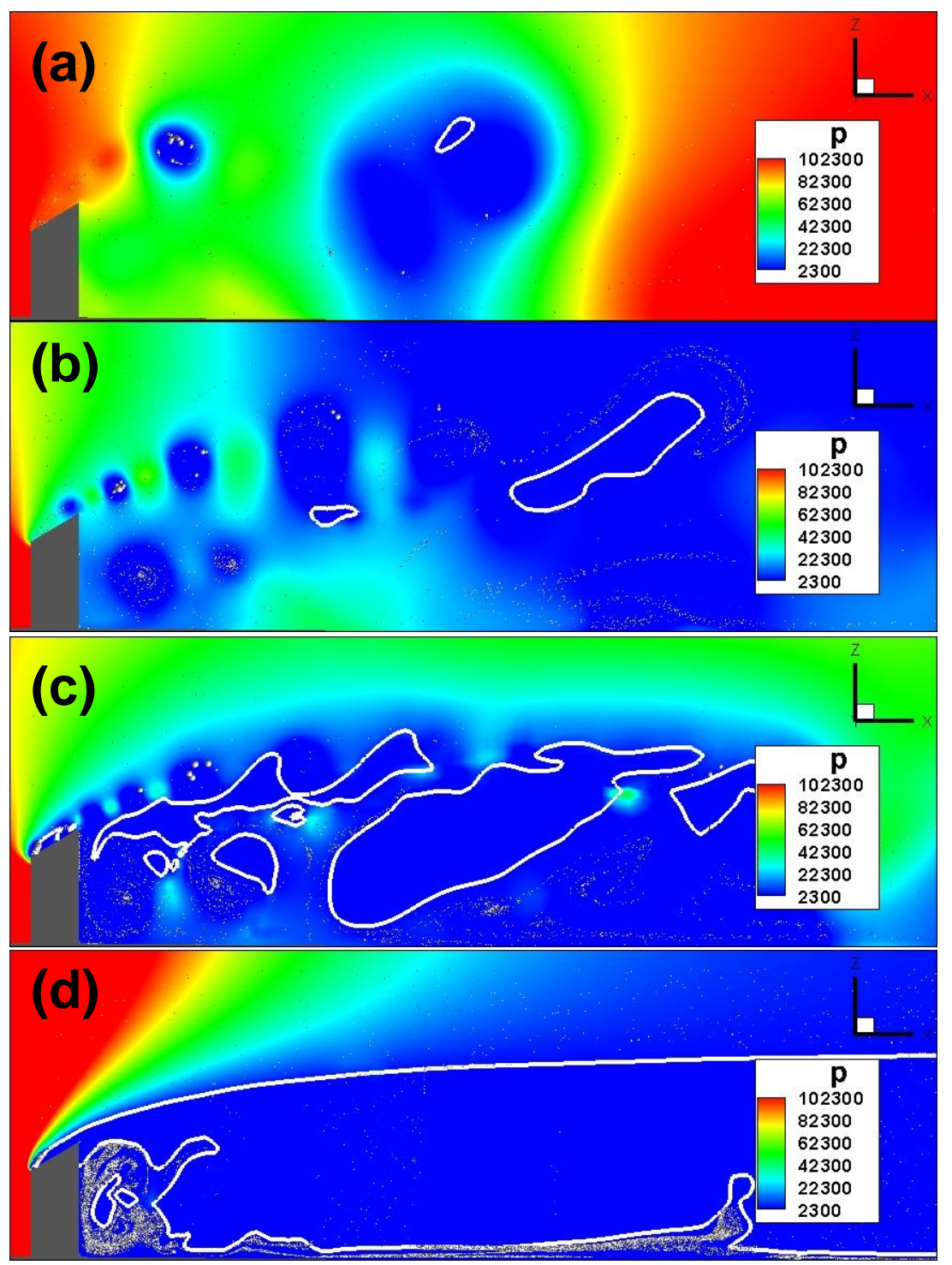

Figure 16. Evolution of the cavitating flow from cavitation inception and limited cavitation to supercavitation under increasing incoming speeds: (a) $6 \mathrm{~m} / \mathrm{s}$; (b) $8 \mathrm{~m} / \mathrm{s}$; (c) $12 \mathrm{~m} / \mathrm{s}$ and (e) 18 $\mathrm{m} / \mathrm{s}$. Color contours represent vorticity. Silver spheres are the nuclei bubbles and the while line correspond to the zero level set cavity interface.

A time sequence of the two-phase flow development behind the body is shown in Figure 15. This highlights nuclei capture and cavitation inception by the multiscale method. In particular, sequences (a) and (b) indicate that the inception can be modeled by enabling the collected bubbles to form an initial cavity. This is achieved with the help of the level set method in the continuum two-phase viscous model. As the bubbles grow beyond a threshold size and/or as bubbles merge, the model initiates a gas-liquid interface dynamics described by the level set method. The initiated cavity continues its growth while elongating (see Figure 15c). 


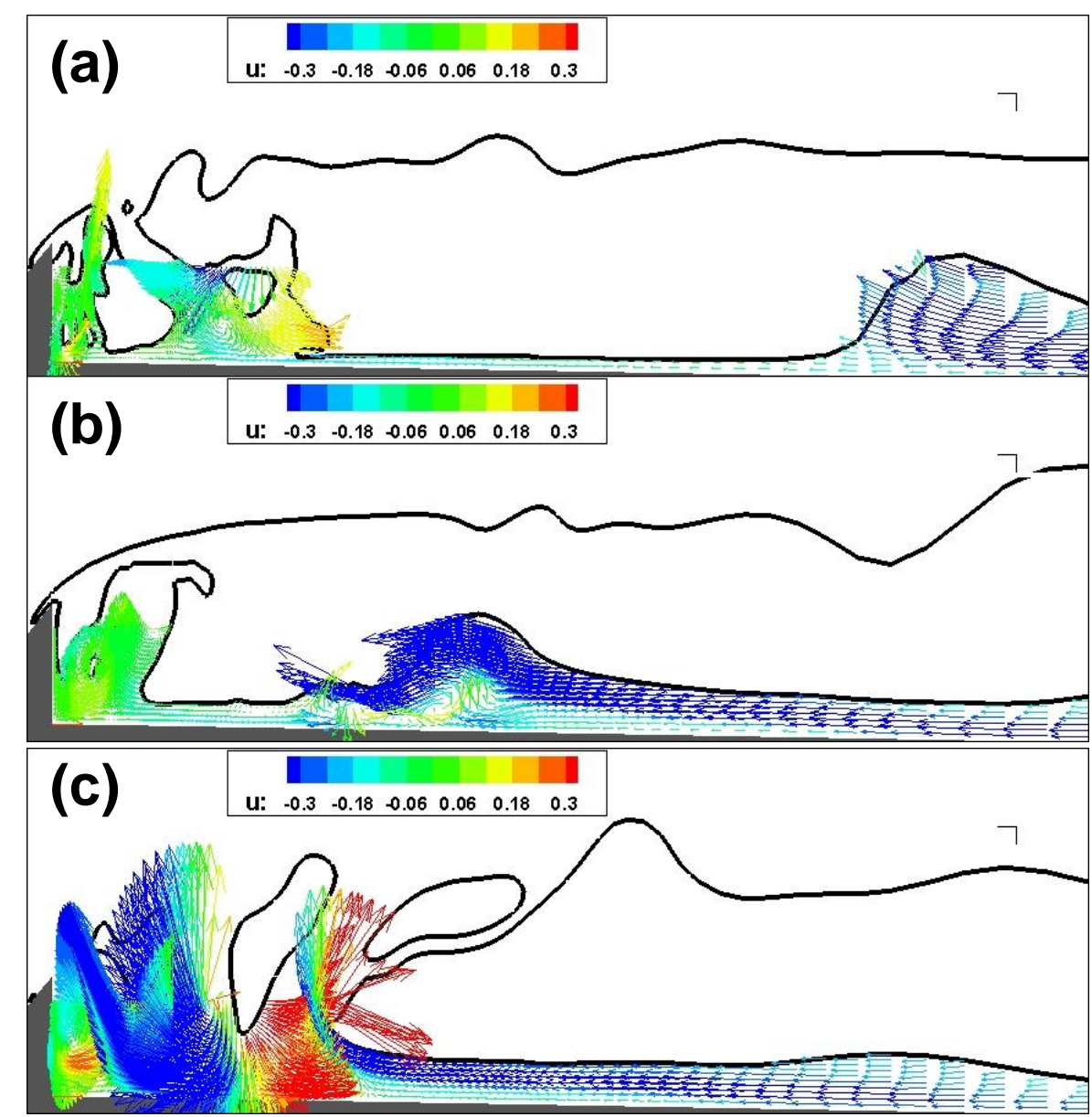

Figure 17. A time sequence of reentrant jet underneath the supercavitation. (The color arrows represent velocity vectors, and the black line corresponds to the cavity interface represented by level zero).

\subsection{Cavitation under Different Flow Velocities}

The pressures on the body and its wake decrease as the flow rate increases. This evolution of the cavitating flow from cavitation inception to limited cavitation and supercavitation is shown in Figure 16. Figure 16a shows at a relatively low flow rate, the shedding vortices carrying downstream bubbles generated from cavitation on the body. With increased flow rate, as shown in Figure 16b, the bubbles generated from the intensified cavitation become denser and the cavities become larger. This trend continues with further increase in the flow rate and partially overlapping cavities start occupying large regions (Figure $16 \mathrm{c}$ ). At the end a supercavity is formed as seen in Figure 16d. The supercavity is not stable and an unsteady reentrant jet enters periodically the cavity opposite to the main flow direction as shown in Figure 16d. This appears as a turbulent mass of liquid moving upstream in the cavity toward the 
projectile. The impact of this reentrant jet on the projectile is strong enough to completely break up the supercavity off the body as seen in Figure 17 which illustrates a sequence of reentrant jet underneath the supercavitation moving towards the body.

\section{Cavitation in Propeller Flow}

The capabilities of developed model for cavitation problems were further extended to deal flows in complex geometries. Fig. 18 illustrates this for the NSWCCD Prop5530 [92-95]. In this simulation bubble nuclei close to the foil surface or the tip vortices locations are seen to become activated by the low pressures. In addition, sheet, cloud, and tip vortex cavitation were captured on the rotating propeller.

To simulate the NSWCCD Prop5530 propeller of diameter $D=0.4 \mathrm{~m}$ (Fig. 18)in a rotational frame, a one-blade-to-blade passage computational domain which follows the incoming flow angle and forms a spiral shape as shown in Fig. 19 is used. The selected computational domain has the inflow boundary located at $R_{p}=D / 2$ and the outflow at $3 R_{p}$ from the propeller mid-plane. The radial domain boundary is located at $2 R_{p}$ from the hub center. Free stream boundary conditions are imposed at inflow, outflow, and radial domain boundaries while the two side (blade-to-blade) boundaries have periodic boundary conditions except at the blade surface where no-slip conditions are imposed.
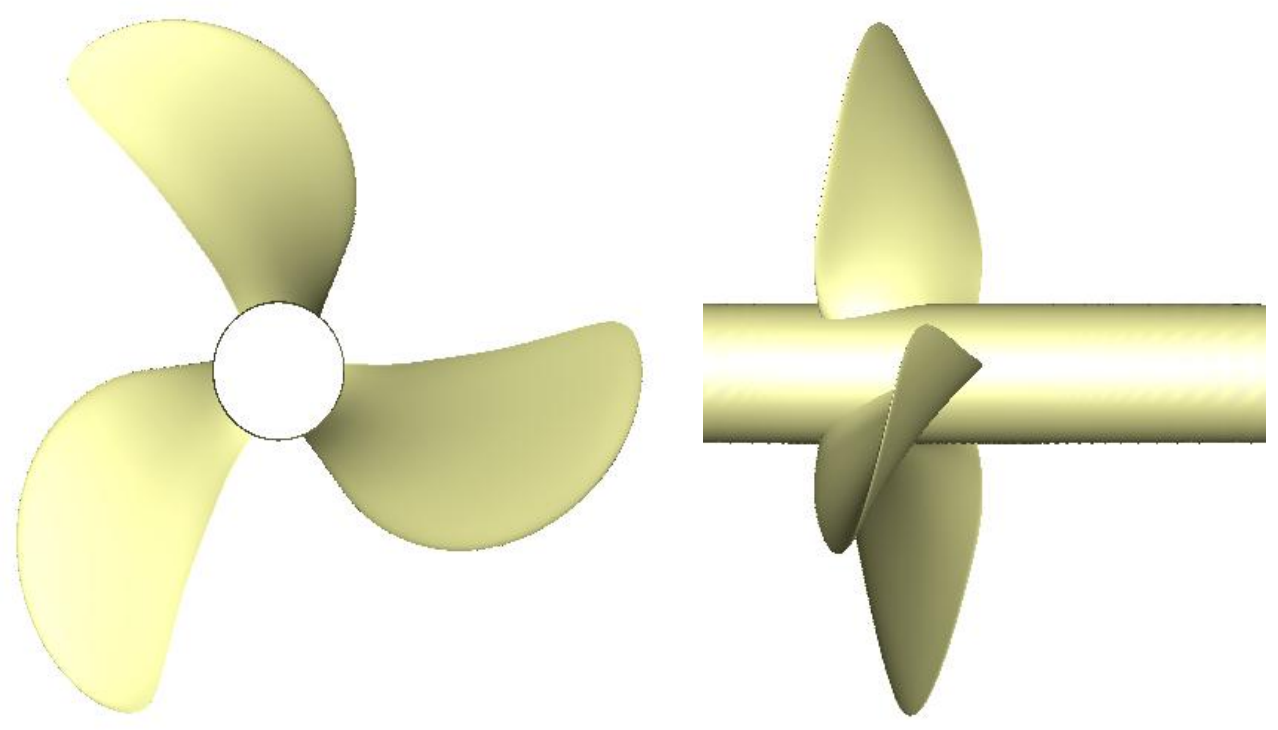

Fig. 18: Geometry of the NSWCCD open propeller 5530. 


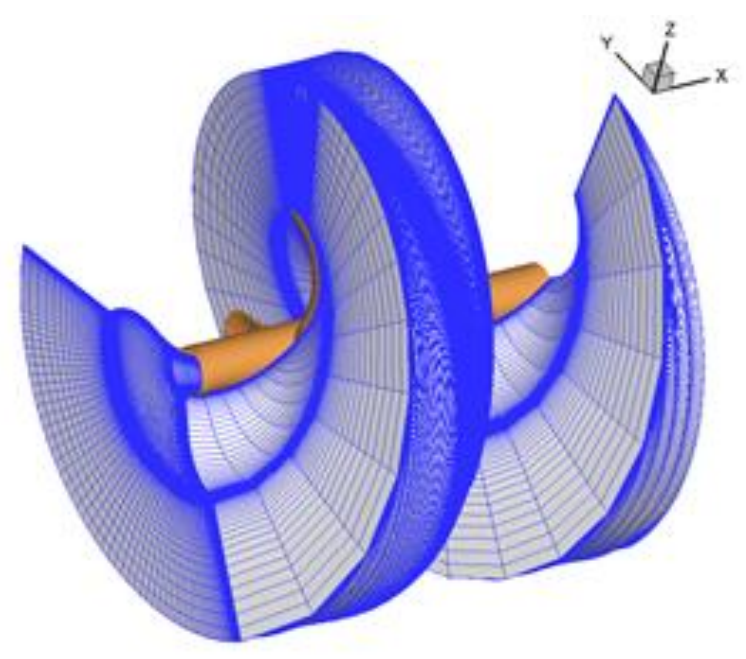

Fig. 19: H-H type grid generated for one blade-to-blade passage of the propeller. A total of 2.1 million grid points for the single passage is used in the calculations.

An H-H type grid with 2.1 million grid points is used to generate the grid for the bladeto-blade computational domain. There are 101 grid points from the blade leading edge to the trailing edge and 81 grid points from the root section to the tip section. Care is taken to ensure that grid points closest to the rigid boundary are at distances of $y^{+} \sim 3$ from the blade surface to properly capture the boundary layer.

The simulation of cavitation on the propeller is started after the single phase flow solution reaches limit cycle oscillation. Nuclei are introduced in the domain from upstream and are also released from the surface of the foil using the solid surface nucleation model. The field nuclei are selected with sizes ranging from $10 \mu \mathrm{m}$ to $50 \mu \mathrm{m}$ and are distributed randomly in the incoming flow field resulting in a void fraction of $1 \times 10^{-6}$. Nucleation from the solid wall is realized with nuclei at size $10 \mu \mathrm{m}$ released initially from the propeller surface when the local pressure drops below vapor pressure. The number of nucleation sites per unit area, $N_{s}$, is selected to be $70 / \mathrm{cm}^{2}$ and the nucleation frequency rate, $f_{n}$, is selected to be $10 \mathrm{kHz}$. These parameters were chosen to minimize the number of nuclei to be tracked in the Lagrangian model without affecting the modeling of the sheet cavity according to our previous parametric study on a 2D hydrofoil case $[87,88]$. Concerning multiscale modeling, the singularity microbubbles are switched into a discretized Level Set free surface when their radius exceeds the local grid size and is larger than $700 \mu \mathrm{m}$ which is about 20 times an initial median nucleus radius.

Fig. 20 shows snapshot images of the sheet cavity at its maximum extent and the cavitating tip vortex for the three advance coefficients at $\sigma=1$. It is seen that the sheet cavity 
extends beyond the trailing edge for all three cases studied. For the $J=1.1$ case the loading is low and the cavitation only occurs near the tip and downstream of the mid-chord of the blade. The circulation in tip vortex is not high enough and trapped bubbles in the viscous core do not grow significantly. As $J$ is reduced, the loading increases and the sheet cavitation extends and moves toward the leading edge; the sheet covers almost the full blade surface for $J=0.9$. In addition, a well-developed tip vortex forms with cavitation bubbles in it and around it for $J=1.0$ and $J=0.9$.
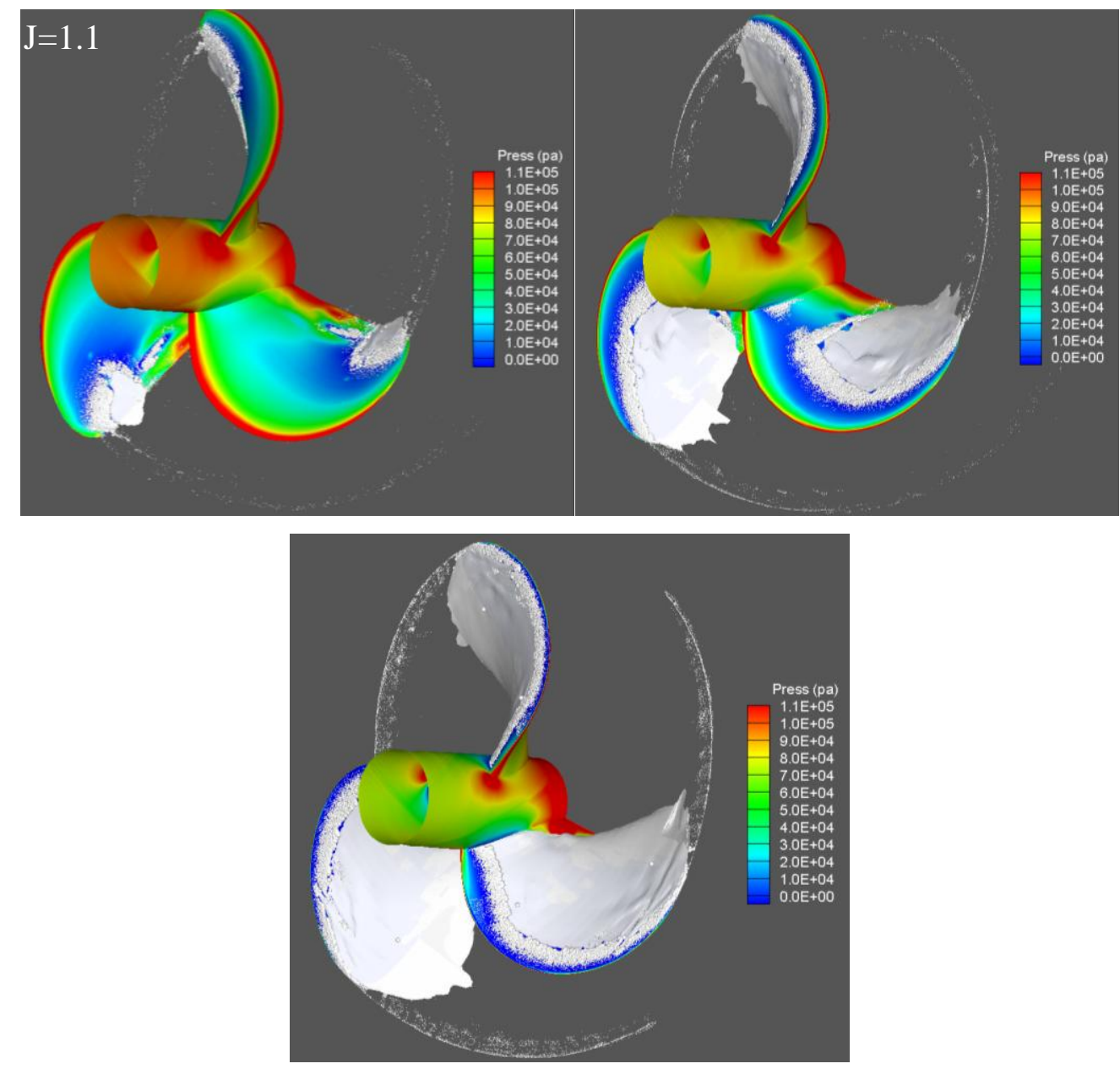

Fig. 20: Snapshots showing sheet cavitation and tip vortex cavitation for a) $J=1.1, b) J=1.0$, and c) $J=0.9$ at the cavitation number $\sigma=1.0$.

The simulations for three values of the advance coefficient, $J=0.91 .0$ and 1.1, are conducted at a cavitation number $\sigma=\left(P_{\infty}-p_{v}\right) /\left(0.5 \rho U_{\infty}^{2}\right)=1$. This cavitation number is chosen to allow cavitation to occur on the blade surface even for the highest pressure coefficient case, $J=1.1$. For each case, the 
simulations were continued until three rotations were completed by the blade. Sheet cavitation and tip vortex cavitation develop gradually from the field nuclei and from nucleation on the blade surface.
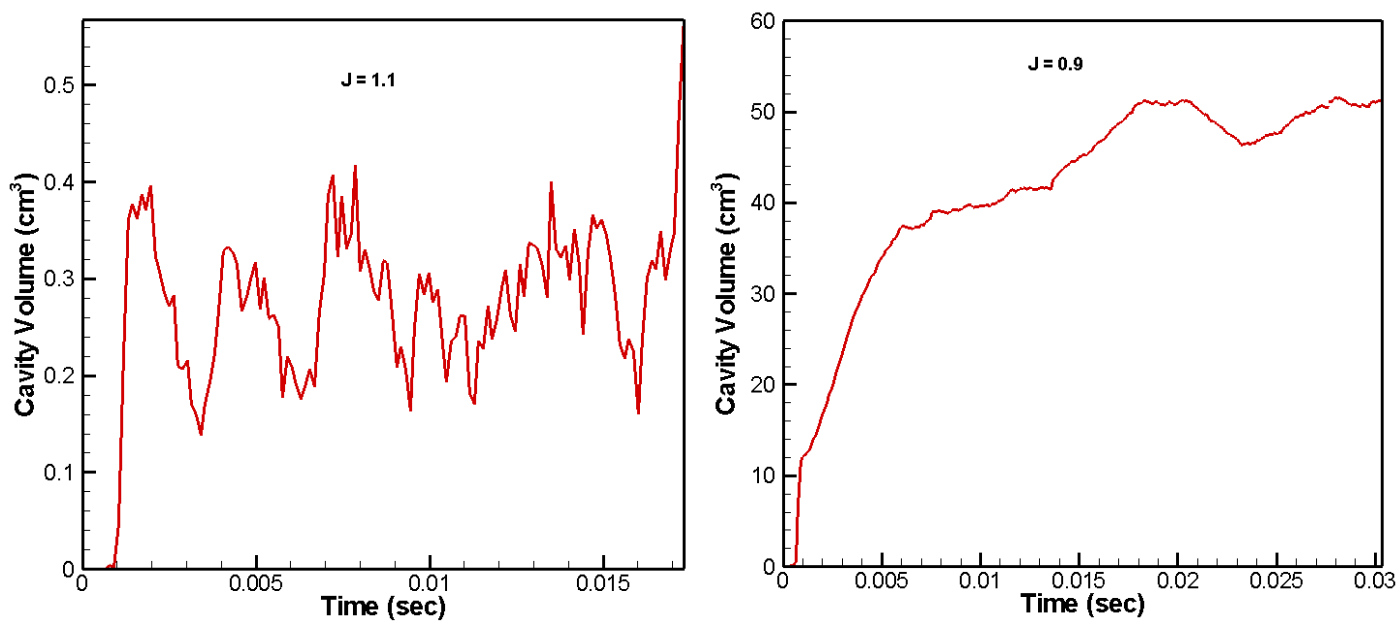

Fig. 21: Sheet cavity volume versus time for $J=1.1$ and 0.9 at cavitation number $\sigma=1.0$.

\section{CONCLUSIONS}

A multi-scale framework was developed for the simulation of various cavitation flows smoothly bridging a Level Set method (LSM) for large size cavities and a Discrete Singularity Model (DSM) for small bubbles. Growth of initially microscopic bubbles treated as singularities and their merger into large discretized cavities was first demonstrated for bubble capture in a Rankine line vortex. Then the model was also applied to simulate nuclei growth and merger into sheet cavitation. Starting with micro-scale physics of nucleation from the solid surface and bulk liquid, sheet cavitation along a NACA0015 hydrofoil is well captured by the model. The predicted sheet lengths and oscillation frequencies under several different cavitation numbers match experimental data very well. Next the simulations of separation and cavitation behind a blunt body were conducted for different flow velocities and corresponding cavitation regimes. When the velocity is relatively small (i.e., large cavitation number), flow separation behind the body results in the shedding of vortices, which capture nuclei in their cores to form elongated vortical cavities. As the flow velocity increases (or as the ambient pressure decreases) the flow evolves into a separated flow with a large cavity behind the body. A reentrant jet may form and move upstream into the cavity towards the body. These results are in good agreements with experimental observations. The method is also applied to simulate various forms of cavitation on 
a rotating propeller including sheet and tip vortex cavitation. The volume of the sheet cavity oscillates in time due to the cavity being sheared off periodically near the trailing edge by the wake flow.

\section{ACKNOWLEDGMENTS}

This work was supported by the Office of Naval Research under contract N00014-12-C0382 monitored by Dr. Ki-Han Kim. This work was also supported in part by grants of computer time from The National Energy Research Scientific Computing Center (NERSC), a scientific computing facility for the Office of Science in the U.S. Department of Energy.

\section{References}

1. Hammitt, F. G. "Cavitation and multiphase flow phenomena, New York, McGraw-Hill, 1980

2. Brennen, C.E., "Hydrodynamics of pumps", Concepts NREC and Oxford University Press, 1994.

3. Chahine, G.L., Kalumuck, K.M. and Frederick, G.S., "Cavitating Water Jets for Deep Hole Drilling in Hard Rock", Proceedings, $8^{\text {th }}$ American Water Jet Conference, Houston, TX, 1995.

4. Kalumuck, K.M. and Chahine, G.L. "The use of cavitating jets to oxidize organic compounds in water", ASME Journal of Fluid Engineering, Vol. 122, pp. 465-470, 2000.

5. Chahine, G.L. and Kalumuck, K.M, "Swirling fluid jet cavitation method and system for efficient decontamination of liquids", US Patent No 6,200,486, assigned to DYNAFLOW INC, 2000.

6. Chahine, G.L. and Kalumuck, K.M, "Fluid jet cavitation method and system for efficient decontamination of liquids", US Patent No 6,221,260, assigned to DYNAFLOW INC, 2001.

7. Kalumuck, K.M., Chahine, G.L., Choi, J.K., Hsiao, C.-T., Aley, P.D., Frederick, G.S. "Development of Cavitating Liquid Jets for Oxidation Remediation of Contaminated Aquifiers", DYNAFLOW INC., Technical Report 2M2022-NIEHS-1, December,2003.

8. Powers, M. "Five Star nanoemulsions enable neutraceuticals as first commercial platform", NanoBiotech News, Vol. 3, pp.1, 2005.

9. Beggs, D.H., Brill, J.P., "A Study of Two-Phase Flow in Inclined Pipes", Journal of Petroleum Technology, Vol.25, No.5, pp.607-617, May 1973.

10. Chahine, G.L., Franc, J.P., Karimi, A., 2013, "Part 1: Cavitation and Cavitation Erosion : Computational and Experimental Approaches" in "Advanced Experimental and Numerical Techniques for Cavitation Erosion Prediction”, Editors K.H. Kim, G.L. Chahine, J.P. Franc, A. Karimi, Springer (to appear).

11. Hsiao, C. T., X. Wu, J. Ma, and G. L. Chahine. 2013. Numerical and experimental study of bubble entrainment due to a horizontal plunging jet. International Shipbuilding Progress 60 (1):435-469. 
12. G.L. Chahine and R. Duraiswami, 1992, "Dynamical Interaction in a multi-Bubble Cloud," Journal of Fluids Engineering, Vol. 114, No. 4, pp. 680-686.

13. Chahine, G. L., 1994, "Strong interactions bubble/bubble and bubble/flow," Bubble Dynamics and Interface Phenomena, edited by J. R. Blake, J. M. Boulton-Stone, and N. H. Thomas Kluwer Academic, Dordrecht, p. 195.

14. Chahine, G.L., Franc, J.P., Karimi, A., 2013 "Advanced Experimental and Numerical Techniques for Cavitation Erosion Prediction - Part II", Editors K.H. Kim, G.L. Chahine, J.P. Franc, A. Karimi, Springer (to appear).

15. Chahine, G.L., Kalumuck, K.M., Tanguay, M., Galambos, J.P., Rayleigh, M., Miller, R.D. and Mairs, C., "Development of a non-explosive ship shock testing system SBIR phase I final report", DYNAFLOW, INC., Technical Report 2M3030-1 DTRC, 2004.

16. Chahine, G.L. and Bovis, A.G., "Pressure field generated by non-spherical bubble collapse", Journal of Fluids Engineeering, Vol. 105, No. 3, pp. 356-364, 1983.

17. Prosperetti, A. and Oguz, H.N., "Physalis: a new $\mathrm{O}(\mathrm{N})$ method for the numerical simulation of disperse systems: potential flow of sphere", Journal of Computational Physics, Vol.167, pp. 196-216, 2001.

18. Fedkiw, R.P., Aslam, T., Merriman, B. and Osher, S., "A non-oscillatory Eulerian approach to interfaces in multimaterial flows (the ghost fluid method)", Journal of Computational Physics, Vol.169, pp. 463-502, 1999.

19. Tryggvason, G, B Bunner, A Esmaeeli, D Juric, N Al-Rawahi, W Tauber, J Han, S Nas, and Y-J Jan. 2001. A front-tracking method for the computations of multiphase flow. Journal of Computational Physics 169 (2):708-759.

20. Osher, S. and Fedkiw, R.P., "Level set methods, an overview and some recent results", Journal of Computational Physics, Vol.169, pp. 463-502, 2001.

21. Fedkiw, R.P., Sapiro, G. and Shu, C.W., "Shock capturing, level sets, and PDE based methods in computer vision and image processing: a review of Osher's contributions", Journal of Computational Physics, Vol.185, pp. 309-341, 2003.

22. Tryggvason, Gretar, Siju Thomas, Jiacai Lu, and Bahman Aboulhasanzadeh. 2010. Multiscale issues in DNS of multiphase flows. Acta Mathematica Scientia 30 (2):551-562.

23. Esmaeeli, A. and Tryggvason, G., 1998, "Direct numerical simulations of bubbly flows. Part 1. Low Reynolds number array", Journal of Fluid Mechanics, 377, pp. 313-345.

24. Oweis, G., van der Hout, I., Iyer, C., Tryggvason, G., and Ceccio, S., 2005, "Capture and inception of bubbles near line vortices," Physics of Fluids, 17, p. 022105.

25. Lu, J., Biswas, S., Tryggvason, G, 2006, "A DNS study of laminar bubbly flows in a vertical channel" International Journal of Multiphase Flow, 32, pp.643-660

26. Seo, J.H, Lele, S. K., Tryggvason, G, 2010, "Investigation and modeling of bubble-bubble interaction effect in homogeneous bubbly flows", Physics of Fluids, 22, pp. 063302

27. Lahey, Richard T. 2009. On the direct numerical simulation of two-phase flows. Nuclear Engineering and Design 239 (5):867-879

28. Bolotnov, I.A., Lahey, R.T., Jr., Drew, D.A., Jansen, K.E. andOberai, A.A. 2010, "Spectral Analysis of Turbulence Based on the DNS of a Channel Flow", Computers \& Fluids, 39, pp. 640-655.

29. Rodriguez, Joseph M, Onkar Sahni, Richard T Lahey, and Kenneth E Jansen. 2013. A parallel adaptive mesh method for the numerical simulation of multiphase flows. Computers $\&$ Fluids. 
30. Xiong, Qingang, Bo Li, and Ji Xu. 2013. GPU-accelerated adaptive particle splitting and merging in SPH. Computer Physics Communications.

31. Ma, Jingsen, Wei Ge, Qingang Xiong, Junwu Wang, and Jinghai Li. 2009. Direct numerical simulation of particle clustering in gas-solid flow with a macro-scale particle method. Chemical Engineering Science 64 (1):43-51.

32. Xiong, Qingang, Bo Li, Feiguo Chen, Jingsen Ma, Wei Ge, and Jinghai Li. 2010. Direct numerical simulation of sub-grid structures in gas-solid flow-GPU implementation of macro-scale pseudo-particle modeling. Chemical Engineering Science 65 (19):5356-5365.

33. Quan, Shaoping, Jing Lou, and David P. Schmidt. 2009. Modeling merging and breakup in the moving mesh interface tracking method for multiphase flow simulations. Journal of Computational Physics 228 (7):2660-2675.

34. Choi, Jin-Keun, and Georges L Chahine. Numerical Study on the Behavior of Air Layers Used for Drag Reduction.

35. Zhang, D.Z. and Prosperetti, A., "Averaged equations for inviscid disperse two-phase flow", Journal of Fluid Mechanics, Vol. 267, pp 185, 1994.

36. Zhang, D.Z. and Prosperetti, A., "Ensemble phase-averaged equations for bubbly flows", Physics of Fluids, Vol. 6, pp. 2956-2970, 1994.

37. Ma, Jingsen, Assad A Oberai, Mark C Hyman, Donald A Drew, and Richard T Lahey Jr. 2011. Two-fluid modeling of bubbly flows around surface ships using a phenomenological subgrid air entrainment model. Computers \& Fluids 52:50-57.

38. Xiang, M, SCP Cheung, JY Tu, and WH Zhang. 2011. Numerical research on drag reduction by ventilated partial cavity based on two-fluid model. Ocean Engineering 38 (17):2023-2032.

39. Biesheuvel, A. and van Wijngaarden, L. "Two-phase flow equations for a dilute dispersion of gas bubble in liquid", Journal of Fluid Mechanics, Vol. 148, pp. 301-318, 1984.

40. Kubota, A., Hiroharu, K., Yamaguchi, H., "A new modeling of cavitating flows: a numerical study of unsteady cavitation on a hydrofoil section", Journal of Fluid Mechanics, Vol. 240, pp. 59-96, 1992.

41. Delale, C.F., Schnerr, G.H., Sauer, J., “Quasi-one-dimensional steady-state cavitating nozzle flows", Journal of Fluid Mechanics, Vol. 427, pp. 167-204, 2001.

42. Hinch, E.J., "An averaged-equation approach to particle interactions in a fluid suspension", Journal of Fluid Mechanics, Vol. 83, pp. 695, 1977.

43. Nigmatulin, R.I., "Spatial averaging in the mechanics of heterogeneous and disperse systems", International Journal of Multiphase Flow, Vol. 5, pp.353-385, 1979.

44. Pauchon, C. and Smereka, P., "Momentum interactions in dispersed flow: an averaging and a variational approach", International Journal of Multiphase Flow, Vol. 18, pp.65-87, 1992.

45. Chen, Y. and Heister, S.D., "Modeling hydrodynamic non-equilibrium in cavitating flows", Journal of Fluids Engineering, Vol. 118, pp.172-178, 1996.

46. Atkin, R.J and Craine, R.E., "Continuum theories of mixtures: basic theory and historical development", Quarterly Journal of Mechanics and Applied Mathematics, Vol. 29, pp.209244, 1976.

47. Atkin, R.J and Craine, R.E., "Continuum theories of mixtures: applications", Journal of the Institute of Mathematics and its Applications, Vol.17, pp. 153-207, 1976.

48. Bown, R.M., "Theory of mixtures", in Continuum Physics, Vol. 3, Academic Press, New York, 1971.

49. Rajagopal, K.R., Tao, L., Mechanics of Mixtures, World Scientific, Singapore, 1995. 
50. Druzhinin, O.A. and Elghobashi, S., "Direct numerical simulations of bubble-laden turbulent flows using the two-fluid formulation", Physics of Fluids, Vol. 10, pp. 685-607, 1998.

51. Druzhinin, O.A. and Elghobashi, S., "A Lagrangian-Eulerian mapping solver for direct numerical simulation of bubble-laden turbulent shear flows using the two-fluid formulation", Journal of Computational Physics, Vol. 154, pp. 174-196, 1999.

52. Druzhinin, O.A. and Elghobashi, S., "Direct numerical simulation of a three-dimensional spatially developing bubble-laden mixing layer with two-way coupling", Journal of Fluid Mechanics, Vol. 429, pp. 23-62, 2001.

53. Elghobashi,S. and Truesdell, G.C., "Direct simulation of particle dispersion in a decaying isotropic turbulence," Journal of Fluid Mechanics, Vol. 242, pp.655-700, 1992.

54. Elghobashi, S. and Truesdell, G.C., "On the two-way interaction between homogeneous turbulence and dispersed solid particle. I: Turbulence modification", Physics of Fluids A: Fluid Dynamics, Vol. 5, pp. 1790-1801, 1993.

55. Ferrante, A. and Elghobashi, S., "On the mechanisms of drag reduction in a spatially developing turbulent boundary layer laden with microbubbles", Journal of Fluid Mechanics, Vol.503, pp. 345-355, 2004.

56. $\mathrm{Xu}$, J., Maxey, M.R. and Karniadakis, G.E., "Numerical simulation of turbulent drag reduction using micro-bubbles", Journal of Fluid Mechanics, Vol. 468, pp. 271-281, 2002.

57. Hsiao, C.-T., Chahine, G.L., "Prediction of Vortex Cavitation Inception Using Coupled Spherical and Non-Spherical Models and Navier-Stokes Computations", Journal of Marine Science and Technology, Vol. 8(3), pp.99-108, 2003.

58. Choi, J.-K., Hsiao, C.-T., Chahine, G.L., "Tip Vortex Cavitation Inception Study Using the Surface Averaged Pressure (SAP) Model Combined with a Bubble Splitting Model”, Proc. 25th Symposium on Naval Hydrodynamics, St. John's, NL, Canada, Aug. 2004.

59. Hsiao, C.-T., Chahine, G.L., Liu, H., "Scaling Effects on Prediction of Cavitation Inception in a Line Vortex Flow", Journal of Fluid Engineering, Vol. 125, pp. 53-60, 2003.

60. Hsiao, C.-T., Jain, A. and Chahine G.L., "Effects of Gas Diffusion on Bubble Entrainment and Dynamics around a Propeller", 26th Symposium on Naval Hydrodynamics, Rome, Italy, 2006.

61. Li, Jinghai, Wei Ge, Wei Wang, and Ning Yang. 2010. Focusing on the meso-scales of multi-scale phenomena-In search for a new paradigm in chemical engineering. Particuology 8 (6):634-639.

62. Li, Jinghai, Wenlai Huang, Peter P Edwards, Mooson Kwauk, John T Houghton, and Daniel Slocombe. 2013. On the Universality of Mesoscience: Science of'the in-between'. arXiv preprint arXiv:1302.5861.

63. Tomiyama, A., Shimada, N., 2001. (N+2)-field modeling for bubbly flow simulation. Comput. Fluid Dyn. J. 9 (4), 418-426.

64. Xiang, M., Cheung, S. C. P., Tu, J. Y., \& Zhang, W. H. 2014. A multi-fluid modelling approach for the air entrainment and internal bubbly flow region in hydraulic jumps. Ocean Engineering, 91, 51-63.

65. Yan, Kai, and Defu Che. 2010. A coupled model for simulation of the gas-liquid two-phase flow with complex flow patterns. International Journal of Multiphase Flow 36 (4):333-348.

66. Tomar, Gaurav, Daniel Fuster, Stéphane Zaleski, and Stéphane Popinet. 2010. Multiscale simulations of primary atomization. Computers \& Fluids 39 (10):1864-1874 
67. Hänsch, Susann, Dirk Lucas, Eckhard Krepper, and Thomas Höhne. 2012. A multi-field two-fluid concept for transitions between different scales of interfacial structures. International Journal of Multiphase Flow.

68. Ma, Jingsen, Assad A Oberai, Richard T Lahey Jr, and Donald A Drew. 2011. Modeling air entrainment and transport in a hydraulic jump using two-fluid RANS and DES turbulence models. Heat and mass transfer 47 (8):911-919.

69. Michael, Thad Jefferson. 2013. Development and validation of a sharp interface cavitation model. PhD Thesis, Iowa University

70. Hsiao, C.-T., Chahine, G.L., "Prediction of Vortex Cavitation Inception Using Coupled Spherical and Non-Spherical Models and Navier-Stokes Computations", Journal of Marine Science and Technology, Vol. 8(3), pp.99-108, 2003.

71. Choi, J., Hsiao, C.-T., Chahine, G.L., Ceccio, S., "Growth, Oscillation and Collapse of Vortex Cavitation Bubbles," Journal of Fluid Mechanics, Vol. 624, pp. 255, 2009.

72. Ma J, Chahine G L and Hsiao C-T, "Spherical bubble dynamics in a bubbly medium using an Euler-Lagrange model", Chemical Engineering Science vol 128 pp 64-81, 2015

73. Chorin, A.J., A numerical method for solving incompressible viscous flow problems. Journal of computational physics, 1967. 2(1): p. 12-26.

74. Roe, P.L., "Approximate Riemann Solvers, Parameter Vectors, and Difference Schemes," Journal of Computational Physics, Vol. 43, pp. 357-372, 1981.

75. van Leer, B., and Woodward, P.R., "The MUSCL Code for Compressible Flow: Philosophy and Results”, B van Leer, PR Woodward - Proc. of the TICOM Conf., Austin, TX, 1979.

76. Vanden, K.J., and Whitfield, D.L., "Direct and Iterative Algorithms for the Three-Dimensional Euler Equations", AIAA Journal, Vol. 33, No. 5, pp. 851-858, 1995.

77. Fedkiw, R.P., et al., A non-oscillatory Eulerian approach to interfaces in multimaterial flows (the ghost fluid method). Journal of Computational Physics, 1999. 152(2): p. 457-492.

78. Sussman, M., et al., An improved level set method for incompressible two-phase flows. Computers \& Fluids, 1998. 27(5): p. 663-680.

79. Carrica, P., R. Wilson, and F. Stern, An unsteady single-phase level set method for viscous free surface flows. International Journal for Numerical Methods in Fluids, 2007. 53(2): p. 229-256.

80. Saffman, P., The lift on a small sphere in a slow shear flow. Journal of Fluid Mechanics, 1965. 22(02): p. 385-400.

81. Singh, S., Fourmeau, T., Choi, J.-K., Chahine, G.L., Thrust Enhancement through Bubble Injection into an Expanding-Contracting Nozzle with a Throat, Journal of Fluids Engineering, 136 (7), Jul. 2014.

82. Choi, J.-K., Wu, X., Chahine, G.L., Bubble Augmented Propulsion with a ConvergentDivergent Nozzle, 30th Symposium on Naval Hydrodynamics, Hobart, Tasmania, Australia, November 2-7, 2014.

83. Harvey, E.N., et al., Bubble formation in animals. II. Gas nuclei and their distribution in blood and tissues. Journal of Cellular and Comparative Physiology, 1944. 24(1): p. 23-34.

84. Mørch, K.A., Cavitation Nuclei: Experiments and Theory. Journal of Hydrodynamics, Ser. B, 2009. 21(2): p. 176-189.

85. Briggs, L.J., Limiting negative pressure of water. Journal of Applied Physics, 2004. 21(7): p. 721-722.

86. Atchley, A.A. and A. Prosperetti, The crevice model of bubble nucleation. The Journal of the Acoustical Society of America, 1989. 86(3): p. 1065-1084. 
87. Hsiao, C.-T., Ma, J., and Chahine, G.L. 2014, "Multiscale Tow-Phase Flow Modeling of Sheet and Cloud Cavitation". 30th Symposium on Naval Hydrodynamics (Hobart, Tasmania, Australia)

88. Hsiao, C.-T., Ma, J., and Chahine, G.L. 2016, "Multiscale Tow-Phase Flow Modeling of Sheet and Cloud Cavitation". Submitted to International Journal of Multiphase Flows

89. Berntsen, G.S., M. Kjeldsen, and R.E. Arndt. Numerical modeling of sheet and tip vortex cavitation with FLUENT 5. in Fourth International Symposium on Cavitation. 2001. Pasadena, CA: NTNU.

90. Wu, X. and Chahine, G., 2007 Characterization of the Content of the Cavity Behind a HighSpeed Supercavitating Body, ASME Journal of Fluids Engineering, 129, pp. 136-145

91. Ma, J., Hsiao, C.-T., and Chahine, G.L., 2016, "Modeling Separation and Cavitation behind a Blunt Body", ASME 2016 Fluids Engineering Division Summer Meeting, Washington, DC, July $10-14,2016$

92. Shen, Y. and P. Dimotakis. The influence of surface cavitation on hydrodynamic forces. in American Towing Tank Conference, 22nd. 1989.

93. Kim, S. and S. Schroeder. Numerical study of thrust-breakdown due to cavitation on a hydrofoil, a propeller, and a waterjet. in Proceedings of the 28th Symposium on Naval Hydrodynamics. 2010.

94. Kim, S.-E. and S. Brewton. A multiphase approach to turbulent cavitating flows. in Proceedings of 27th Symposium on Naval Hydrodynamics, Seoul, Korea. 2008.

95. Forero M 2010 An investigation on the hydrodynamic impact of treating propeller blade surface to resist cavitation erosion (NSWCCD-50-TR-2010/023) 